\title{
NONLINEAR WAVE EQUATIONS WITH NONLINEAR TRANSMISSION ACOUSTIC CONDITION
}

\author{
SEVDA E. ISAYEVA
}

\begin{abstract}
In this paper we consider a mixed problem for nonlinear wave equations with nonlinear transmission acoustic condition. We prove a theorem on local existence and uniqueness of solutions for this problem by using the Faedo-Galerkin approximations combined with a contraction mapping theorem.
\end{abstract}

\section{Introduction}

Let $\Omega$ be a bounded domain in $R^{n}(n \geq 1)$ with smooth boundary $\Gamma_{1}, \Omega_{2} \subset \Omega$ be a subdomain with smooth boundary $\Gamma_{2}$ and $\Omega_{1}=\Omega \backslash\left(\Omega_{2} \cup \Gamma_{2}\right)$ be a subdomain with boundary $\Gamma=\Gamma_{1} \cup \Gamma_{2}, \Gamma_{1} \cap \Gamma_{2}=\emptyset$. The nonlinear transmission acoustic problem considered here is

$$
\begin{gathered}
u_{t t}-\Delta u+\left|u_{t}\right|^{q_{1}-1} u_{t}=f(u) \quad \text { in } \Omega_{1} \times(0, \infty), \\
v_{t t}-\Delta v+\left|v_{t}\right|^{q_{2}-1} v_{t}=g(v) \text { in } \Omega_{2} \times(0, \infty), \\
M \delta_{t t}+D \delta_{t}+K \delta=-u_{t} \text { on } \Gamma_{2} \times(0, \infty), \\
u=0 \text { on } \Gamma_{1} \times(0, \infty), \\
u=v, \frac{\partial u}{\partial \nu}-\frac{\partial v}{\partial \nu}+\rho\left(u_{t}\right)=\delta_{t} \quad \text { on } \Gamma_{2} \times(0, \infty), \\
u(x, 0)=u_{0}(x), u_{t}(x, 0)=u_{1}(x), x \in \Omega_{1}, \\
v(x, 0)=v_{0}(x), v_{t}(x, 0)=v_{1}(x), x \in \Omega_{2}, \\
\delta(x, 0)=\delta_{0}(x), \delta_{t}(x, 0)=\frac{\partial u_{0}}{\partial \nu}-\frac{\partial v_{0}}{\partial \nu}+\rho\left(u_{1}\right) \equiv \delta_{1}(x), x \in \Gamma_{2},
\end{gathered}
$$

where $\nu$ is the unit outward normal vector to $\Gamma ; f, g, \rho:(-\infty,+\infty) \rightarrow(-\infty,+\infty)$, $M, D, K: \bar{\Gamma}_{2} \rightarrow(-\infty,+\infty), u_{0}, u_{1}: \Omega_{1} \rightarrow(-\infty,+\infty), v_{0}, v_{1}: \Omega_{2} \rightarrow(-\infty,+\infty)$, $\delta_{0}: \Gamma_{2} \rightarrow(-\infty,+\infty)$ are given functions; $q_{i}>1, i=1,2$ are constants.

The problems like (1.1)-(1.8), called transmission acoustic problems, are related to the problem of two wave equations, which models the transverse acoustic vibrations of the membrane composed by two different materials $\Omega_{1}$ and $\Omega_{2}$.

Transmission problems were studied, for example, in $[3,4,5,13,26,28,31]$. The transmission problem to hyperbolic equations was investigated by Dautray and Lions [13] who proved the existence and regularity of solutions for the linear

2010 Mathematics Subject Classification. 35L05, 35L70.

Key words and phrases. Nonlinear wave equation, nonlinear transmission acoustic condition, local solution, contraction mapping theorem. 
problem. Bae [4] studied the transmission problem, in which one component is clamped and the other is in a viscoelastic fluid producing a dissipation mechanism on the boundary, and established a decay result.

Aliev and Mammadhasanov [3] studied the initial boundary value problem on longitudinal impact on a composite linear viscoelastic bar and established a wellposedness result by the method of dynamic regularization of transmission and boundary conditions.

The acoustic boundary conditions were studied in $[6,7,8,9,10,12,14,15,16$, $17,18,19,20,22,23,24,25,29,30,32,33,34]$. A mixed problem for wave equation with nonlinear acoustic boundary conditions was considered by Gao, Liang, Xiao [20], Graber [22, 23]. Graber and Said-Houari [24] studied the stability of a structural acoustic wave equation with semilinear porous acoustic boundary conditions and obtained several results in local existence, global existence, the decay rate and blow up results.

The problems like (1.1)-(1.8) with linear acoustic conditions were studied in $[1,2]$ in which some results in local existence, global existence, the exponential stability and blow up results were obtained.

In this paper we prove the theorem on local existence and uniqueness of solutions for the problem (1.1)-(1.8) by using the Faedo-Galerkin approximations, the compactness method and the fixed point theorem.

Our paper is organized as follows. In section 2 we introduce some notations, preliminaries and statement of well-posedness result for the problem (1.1)-(1.8), which is proved in section 3 .

\section{Preliminaries and main result}

The inner product and norm in $L^{2}\left(\Omega_{i}\right), i=1,2$ and $L^{2}\left(\Gamma_{2}\right)$ are denoted respectively, by

$$
\begin{gathered}
(u, v)_{i}=\int_{\Omega_{i}} u(x) v(x) d x,\|u\|_{i}=\left(\int_{\Omega_{i}}(u(x))^{2} d x\right)^{1 / 2}, i=1,2, \\
(\delta, \theta)_{\Gamma_{2}}=\int_{\Gamma_{2}} \delta(x) \theta(x) d \Gamma_{2},\|\delta\|_{\Gamma_{2}}=\left(\int_{\Gamma_{2}}(\delta(x))^{2} d \Gamma_{2}\right)^{1 / 2} .
\end{gathered}
$$

$H^{1}\left(\Omega_{i}\right), i=1,2$ are the usual real Sobolev spaces of first order. We define a closed subspace of the $H^{1}\left(\Omega_{i}\right)$ as

$$
H_{\Gamma_{1}}^{1}\left(\Omega_{1}\right)=\left\{u \in H^{1}\left(\Omega_{1}\right): \gamma_{0}(u)=0 \text { a.e.on } \Gamma_{1}\right\},
$$

where $\gamma_{0}: H^{1}\left(\Omega_{1}\right) \rightarrow H^{1 / 2}(\Gamma)$ is the trace map of order zero and $H^{1 / 2}(\Gamma)$ is the Sobolev space of order $\frac{1}{2}$ defined over $\Gamma$, as introduced by Lions and Magenes [27]. Observe that the norm in $H_{\Gamma_{1}}^{1}\left(\Omega_{i}\right)$ :

$$
\|u\|_{H_{\Gamma_{1}}^{1}\left(\Omega_{1}\right)}=\left(\sum_{i=1}^{n} \int_{\Omega_{1}}\left(\frac{\partial u}{\partial x_{i}}\right)^{2} d x\right)^{1 / 2}
$$


and the norm of the real Sobolev space $H^{1}\left(\Omega_{i}\right)$ are equivalent, because the Poincaré's inequality holds in $H_{\Gamma_{1}}^{1}\left(\Omega_{i}\right)$. Thus we consider $H_{\Gamma_{1}}^{1}\left(\Omega_{i}\right)$ with the above gradient norm.

The map $\gamma_{1}: H\left(\Delta, \Omega_{1}\right) \cup H\left(\Delta, \Omega_{2}\right) \rightarrow H^{-1 / 2}\left(\Gamma_{2}\right)$ is the Neumann trace map on $H\left(\Delta, \Omega_{1}\right) \cup H\left(\Delta, \Omega_{2}\right)$ and

$$
H\left(\Delta, \Omega_{i}\right)=\left\{u \in H^{1}\left(\Omega_{i}\right): \Delta u \in L^{2}\left(\Omega_{i}\right)\right\}, \quad i=1,2
$$

are equipped with the norms

$$
\|u\|_{\Delta, \Omega_{i}}=\left(\|u\|_{H^{1}\left(\Omega_{i}\right)}^{2}+\|\Delta u\|_{i}^{2}\right)^{1 / 2}, \quad i=1,2 .
$$

The well-posedness result is contained in the following theorem.

Theorem 2.1. Assume that

$$
\begin{gathered}
M, D, K \in C\left(\bar{\Gamma}_{2}\right), M>0, D>0, K>0 \text { for } \forall x \in \bar{\Gamma}_{2} \\
f, g \in C^{1}(-\infty ;+\infty),|f(s)| \leq c_{1}|s|^{p} \\
\left|f^{\prime}(s)\right| \leq c_{2}|s|^{p-1},|g(s)| \leq c_{3}|s|^{p},\left|g^{\prime}(s)\right| \leq c_{4}|s|^{p-1}\left(c_{i}>0, i=1,2,3,4\right) \\
p>1 \text { if } n=1,2,1<p \leq \frac{n}{n-2} \text { if } n \geq 3 \\
\rho \in C^{1}(-\infty ;+\infty),|\rho(s)| \leq c_{5}|s|^{q_{1}}\left(c_{5}>0\right) \\
\rho(s) \text { is monotone increasing function on }(-\infty ;+\infty) \text { with } \rho(0)=0
\end{gathered}
$$

Then for $\forall\left(u_{0}, v_{0}, \delta_{0}\right) \in H_{\Gamma_{1}}^{1}\left(\Omega_{1}\right) \times H^{1}\left(\Omega_{2}\right) \times L^{2}\left(\Gamma_{2}\right), \forall\left(u_{1}, v_{1}, \delta_{1}\right) \in L^{2 q_{1}}\left(\Omega_{1}\right) \times$ $L^{2 q_{2}}\left(\Omega_{2}\right) \times L^{2}\left(\Gamma_{2}\right)\left(\left.u_{0}\right|_{\Gamma_{2}}=\left.v_{0}\right|_{\Gamma_{2}},\left.u_{1}\right|_{\Gamma_{2}}=\left.v_{1}\right|_{\Gamma_{2}}\right)$ there exists $T>0$ such that the problem (1.1)-(1.8) has a unique solution $(u, v, \delta)$ which satisfies

$$
\begin{gathered}
u \in C\left([0, T] ; H_{\Gamma_{1}}^{1}\left(\Omega_{1}\right)\right), u_{t} \in C\left([0, T] ; L^{2}\left(\Omega_{1}\right)\right) \cap L^{q_{1}+1}\left(\Omega_{1} \times(0, T)\right), \\
v \in C\left([0, T] ; H^{1}\left(\Omega_{2}\right)\right), v_{t} \in C\left([0, T] ; L^{2}\left(\Omega_{2}\right)\right) \cap L^{q_{2}+1}\left(\Omega_{2} \times(0, T)\right), \\
\delta, \delta_{t} \in L^{\infty}\left(0, T ; L^{2}\left(\Gamma_{2}\right)\right) .
\end{gathered}
$$

Moreover, if $T_{\max }>0$ is the length of the maximal existence interval of the solution $(u, v, \delta)$, then either $T_{\max }=+\infty$, or

$$
\lim _{t \rightarrow T-0}\left(\left\|u_{t}\right\|_{1}^{2}+\left\|v_{t}\right\|_{2}^{2}+\|\nabla u\|_{1}^{2}+\|\nabla v\|_{2}^{2}+\left\|\sqrt{M} \delta_{t}\right\|_{\Gamma_{2}}^{2}+\|\sqrt{K} \delta\|_{\Gamma_{2}}^{2}\right)=+\infty .
$$

\section{Proof of Theorem 2.1}

We prove this theorem using the combination of the Faedo-Galerkin approximation, the compactness method and the fixed point theorem.

We consider the following problem:

$$
\begin{gathered}
U_{t t}-\Delta U+\left|U_{t}\right|^{q_{1}-1} U_{t}=F \text { in } \Omega_{1} \times(0, T), \\
V_{t t}-\Delta V+\left|V_{t}\right|^{q_{2}-1} V_{t}=G \text { in } \Omega_{2} \times(0, T), \\
M \delta_{t t}+D \delta_{t}+K \delta=-U_{t} \text { on } \Gamma_{2} \times(0, T), \\
U=0 \text { on } \Gamma_{1} \times(0, T), \\
U=V, \frac{\partial U}{\partial \nu}-\frac{\partial V}{\partial \nu}+\rho\left(U_{t}\right)=\delta_{t} \text { on } \Gamma_{2} \times(0, T), \\
U(x, 0)=u_{0}(x), U_{t}(x, 0)=u_{1}(x), x \in \Omega_{1},
\end{gathered}
$$




$$
\begin{aligned}
V(x, 0) & =v_{0}(x), V_{t}(x, 0)=v_{1}(x), x \in \Omega_{2}, \\
\delta(x, 0) & =\delta_{0}(x), \delta_{t}(x, 0)=\delta_{1}(x), x \in \Gamma_{2} ;
\end{aligned}
$$

here $T>0 ; F=F(x, t)$ and $G=G(x, t)$ are fixed functions on $\Omega_{1} \times[0, T)$ and $\Omega_{2} \times[0, T)$, respectively.

To prove Theorem 2.1 we need two lemmas (Lemma 3.1 and Lemma 3.2).

Lemma 3.1. Suppose that (2.1), (2.4)-(2.5) hold and let

$$
\begin{gathered}
F \in H^{1}\left(0, T ; L^{2}\left(\Omega_{1}\right)\right), G \in H^{1}\left(0, T ; L^{2}\left(\Omega_{2}\right)\right), \\
u_{0} \in H_{\Gamma_{1}}^{1}\left(\Omega_{1}\right) \cap H^{2}\left(\Omega_{1}\right), u_{1} \in H_{\Gamma_{1}}^{1}\left(\Omega_{1}\right) \cap L^{2 q_{1}}\left(\Omega_{1}\right), \\
v_{0} \in H^{2}\left(\Omega_{2}\right), v_{1} \in H^{1}\left(\Omega_{2}\right) \cap L^{2 q_{2}}\left(\Omega_{2}\right), \\
\delta_{0}, \delta_{1} \in L^{2}\left(\Gamma_{2}\right) .
\end{gathered}
$$

Then, there exists a unique solution $(U, V, \delta)$ to the problem (3.1)-(3.8) such that

$$
\begin{gathered}
U \in L^{\infty}\left(0, T ; H_{\Gamma_{1}}^{1}\left(\Omega_{1}\right)\right), U_{t} \in L^{\infty}\left(0, T ; H_{\Gamma_{1}}^{1}\left(\Omega_{1}\right)\right) \cap L^{q_{1}+1}\left(\Omega_{1} \times(0, T)\right), \\
U_{t t} \in L^{\infty}\left(0, T ; L^{2}\left(\Omega_{1}\right)\right) \\
V \in L^{\infty}\left(0, T ; H^{1}\left(\Omega_{2}\right)\right), V_{t} \in L^{\infty}\left(0, T ; H^{1}\left(\Omega_{2}\right)\right) \cap L^{q_{2}+1}\left(\Omega_{2} \times(0, T)\right) \\
V_{t t} \in L^{\infty}\left(0, T ; L^{2}\left(\Omega_{2}\right)\right) \\
\left(-\Delta U+\left|U_{t}\right|^{q_{1}-1} U_{t}\right)(t) \in L^{2}\left(\Omega_{1}\right) \\
\left(-\Delta V+\left|V_{t}\right|^{q_{2}-1} V_{t}\right)(t) \in L^{2}\left(\Omega_{2}\right) \text { a.e. on } \quad(0, T) \\
\delta, \delta_{t}, \delta_{t t} \in L^{\infty}\left(0, T ; L^{2}\left(\Gamma_{2}\right)\right) .
\end{gathered}
$$

Proof of Lemma 3.1. Faedo-Galerkin approximation. Let $\left\{\left(\Phi_{j}, \Psi_{j}, e_{j}\right)\right\}$ $(j \in N)$ be orthonormal basis in $W=\left\{(u, v, \delta) \in H_{\Gamma_{1}}^{1}\left(\Omega_{1}\right) \times H^{1}\left(\Omega_{2}\right) \times L^{2}\left(\Gamma_{2}\right)\right.$, $\left.\left.u\right|_{\Gamma_{2}}=\left.v\right|_{\Gamma_{2}}\right\}$. Since $\Gamma_{1}$ and $\Gamma_{2}$ are sufficiently smooth, we have that $\Phi_{j} \in$ $H_{\Gamma_{1}}^{1}\left(\Omega_{1}\right) \cap L^{\infty}\left(\Omega_{1}\right)$ and $\Psi_{j} \in H^{1}\left(\Omega_{2}\right) \cap L^{\infty}\left(\Omega_{2}\right)$ for all $j \in N$. For each $m \in N$ we consider

$$
U_{m}: \Omega_{1} \times\left[0, T_{m}\right] \rightarrow R, V_{m}: \Omega_{2} \times\left[0, T_{m}\right] \rightarrow R, \delta_{m}: \Gamma_{2} \times\left[0, T_{m}\right] \rightarrow R
$$

defined by

$$
\begin{gathered}
U_{m}(x, t)=\sum_{j=1}^{m} \alpha_{j m}(t) \Phi_{j}(x), V_{m}(x, t)=\sum_{j=1}^{m} \beta_{j m}(t) \Psi_{j}(x), \\
\delta_{m}(x, t)=\sum_{j=1}^{m} \eta_{j m}(t) e_{j}(x),
\end{gathered}
$$

which are solutions to the approximate problem :

$$
\begin{gathered}
\left(U_{m t t}, \Phi_{j}\right)_{1}+\left(\nabla U_{m}, \nabla \Phi_{j}\right)_{1}- \\
-\left(\frac{\partial U_{m}}{\partial \nu}, \gamma_{0}\left(\Phi_{j}\right)\right)_{\Gamma_{2}}+\left(\left|U_{m t}\right|^{q_{1}-1} U_{m t}, \Phi_{j}\right)_{1}=\left(F, \Phi_{j}\right)_{1}, \\
\left(V_{m t t}, \Psi_{j}\right)_{2}+\left(\nabla V_{m}, \nabla \Psi_{j}\right)_{2}+ \\
+\left(\frac{\partial V_{m}}{\partial \nu}, \gamma_{0}\left(\Psi_{j}\right)\right)_{\Gamma_{2}}+\left(\left|V_{m t}\right|^{q_{2}-1} V_{m t}, \Psi_{j}\right)_{2}=\left(G, \Psi_{j}\right)_{2}, \\
\left(M \delta_{m t t}+D \delta_{m t}+K \delta_{m}, e_{j}\right)_{\Gamma_{2}}=-\left(\gamma_{0}\left(U_{m t}\right), e_{j}\right)_{\Gamma_{2}},
\end{gathered}
$$




$$
\begin{gathered}
U_{m}=V_{m}, \frac{\partial U_{m}}{\partial \nu}-\frac{\partial V_{m}}{\partial \nu}+\rho\left(U_{m t}\right)=\delta_{m t}, x \in \Gamma_{2}, \\
U_{m}(x, 0)=U_{0 m}(x)=\sum_{j=1}^{m}\left(u_{0}, \Phi_{j}\right)_{1} \Phi_{j}, U_{m_{t}}(x, 0)=U_{1 m}(x)=\sum_{j=1}^{m}\left(u_{1}, \Phi_{j}\right)_{1} \Phi_{j}, \\
V_{m}(x, 0)=V_{0 m}(x)=\sum_{j=1}^{m}\left(v_{0}, \Psi_{j}\right)_{2} \Psi_{j}, V_{m_{t}}(x, 0)=V_{1 m}(x)=\sum_{j=1}^{m}\left(v_{1}, \Psi_{j}\right)_{2} \Psi_{j}, \\
\delta_{m}(x, 0)=\delta_{0 m}(x)=\sum_{j=1}^{m}\left(\delta_{0}, e_{j}\right)_{\Gamma_{2}} e_{j}, \\
\delta_{m_{t}}(x, 0)=\gamma_{1}\left(U_{0 m}-V_{0 m}\right)+\gamma_{0}\left(\rho\left(U_{1 m}\right)\right)=\sum_{j=1}^{m}\left(\gamma_{1}\left(u_{0}-v_{0}\right)+\gamma_{0}\left(\rho\left(u_{1}\right)\right), e_{j}\right)_{\Gamma_{2}} e_{j} .
\end{gathered}
$$

The local existence of such solutions $\left(U_{m}, V_{m}, \delta_{m}\right), m \in N$ of this problem on the interval $\left[0, T_{m}\right]$ is obvious. From $(3.17)-(3.19)$ we have the approximate equations

$$
\begin{gathered}
\left(U_{m t t}, \Phi\right)_{1}+\left(\nabla U_{m}, \nabla \Phi\right)_{1}- \\
-\left(\frac{\partial U_{m}}{\partial \nu}, \gamma_{0}(\Phi)\right)_{\Gamma_{2}}+\left(\left|U_{m t}\right|^{q_{1}-1} U_{m t}, \Phi\right)_{1}=(F, \Phi)_{1} \\
\left(V_{m t t}, \Psi\right)_{2}+\left(\nabla V_{m}, \nabla \Psi\right)_{2}+ \\
+\left(\frac{\partial V_{m}}{\partial \nu}, \gamma_{0}(\Psi)\right)_{\Gamma_{2}}+\left(\left|V_{m t}\right|^{q_{2}-1} V_{m t}, \Psi\right)_{2}=(G, \Psi)_{2} \\
\left(M \delta_{m t t}+D \delta_{m_{t}}+K \delta_{m}, e\right)_{\Gamma_{2}}=-\left(\gamma_{0}\left(U_{m t}\right), e\right)_{\Gamma_{2}}
\end{gathered}
$$

for $\forall \Phi \in \operatorname{Span}\left\{\Phi_{1}, \Phi_{2}, \ldots, \Phi_{m}, \ldots\right\}, \forall \Psi \in \operatorname{Span}\left\{\Psi_{1}, \Psi_{2}, \ldots, \Psi_{m}, \ldots\right\}$, $\forall e \in \operatorname{Span}\left\{e_{1}, e_{2}, \ldots, e_{m}, \ldots\right\}$.

Estimate 1. Taking $\Phi=2 U_{m t}$ in (3.21), $\Psi=2 V_{m t}$ in (3.22), $e=2 \delta_{m t}$ in (3.23), we find

$$
\begin{gathered}
\frac{d}{d t}\left\|U_{m_{t}}\right\|_{1}^{2}+\frac{d}{d t}\left\|\nabla U_{m}\right\|_{1}^{2}-\left(\frac{\partial U_{m}}{\partial \nu}, \gamma_{0}\left(2 U_{m_{t}}\right)\right)_{\Gamma_{2}}+ \\
+2\left(\left|U_{m_{t}}\right|^{q_{1}-1} U_{m_{t}}, U_{m_{t}}\right)_{1}=2\left(F, U_{m_{t}}\right)_{1} \\
\frac{d}{d t}\left\|V_{m_{t}}\right\|_{2}^{2}+\frac{d}{d t}\left\|\nabla V_{m}\right\|_{2}^{2}+\left(\frac{\partial V_{m}}{\partial \nu}, \gamma_{0}\left(2 V_{m_{t}}\right)\right)_{\Gamma_{2}}+ \\
+2\left(\left|V_{m_{t}}\right|^{q_{2}-1} V_{m_{t}}, V_{m_{t}}\right)_{2}=2\left(G, V_{m_{t}}\right)_{2}, \\
\frac{d}{d t}\left\|\sqrt{M} \delta_{m_{t}}\right\|_{\Gamma_{2}}^{2}+2\left\|\sqrt{D} \delta_{m_{t}}\right\|_{\Gamma_{2}}^{2}+\frac{d}{d t}\left\|\sqrt{K} \delta_{m}\right\|_{\Gamma_{2}}^{2}+\left(\gamma_{0}\left(U_{m_{t}}\right), 2 \delta_{m_{t}}\right)_{\Gamma_{2}}=0
\end{gathered}
$$

whence using (3.20), we obtain

$$
\begin{gathered}
\frac{d}{d t}\left(\left\|U_{m_{t}}\right\|_{1}^{2}+\left\|\nabla U_{m}\right\|_{1}^{2}+\left\|V_{m_{t}}\right\|_{2}^{2}+\left\|\nabla V_{m}\right\|_{2}^{2}+\left\|\sqrt{M} \delta_{m_{t}}\right\|_{\Gamma_{2}}^{2}+\left\|\sqrt{K} \delta_{m}\right\|_{\Gamma_{2}}^{2}\right)+ \\
+2\left(\rho\left(U_{m_{t}}\right), U_{m_{t}}\right)_{\Gamma_{2}}+2\left\|\sqrt{D} \delta_{m_{t}}\right\|_{\Gamma_{2}}^{2}+2\left(\left|U_{m_{t}}\right|^{q_{1}+1}, 1\right)_{1}+2\left(\left|V_{m_{t}}\right|^{q_{2}+1}, 1\right)_{2}= \\
=2\left(F, U_{m_{t}}\right)_{1}+2\left(G, V_{m_{t}}\right)_{2}
\end{gathered}
$$


or since by (2.5): $\rho(s) s \geq 0, \forall s \in(-\infty,+\infty)$, we have

$$
\begin{gathered}
\frac{d}{d t}\left(\left\|U_{m_{t}}\right\|_{1}^{2}+\left\|\nabla U_{m}\right\|_{1}^{2}+\left\|V_{m_{t}}\right\|_{2}^{2}+\left\|\nabla V_{m}\right\|_{2}^{2}+\left\|\sqrt{M} \delta_{m_{t}}\right\|_{\Gamma_{2}}^{2}+\left\|\sqrt{K} \delta_{m}\right\|_{\Gamma_{2}}^{2}\right)+ \\
+2\left\|\sqrt{D} \delta_{m_{t}}\right\|_{\Gamma_{2}}^{2}+2\left(\left|U_{m_{t}}\right|^{q_{1}+1}, 1\right)_{1}+2\left(\left|V_{m_{t}}\right|^{q_{2}+1}, 1\right)_{2}= \\
=2\left(F, U_{m_{t}}\right)_{1}+2\left(G, V_{m_{t}}\right)_{2} .
\end{gathered}
$$

Integrating this from 0 to $t\left(t \leq T_{m}\right)$ and using Young inequality, we get

$$
\begin{gathered}
\left\|U_{m_{t}}\right\|_{1}^{2}+\left\|\nabla U_{m}\right\|_{1}^{2}+\left\|V_{m_{t}}\right\|_{2}^{2}+\left\|\nabla V_{m}\right\|_{2}^{2}+\left\|\sqrt{M} \delta_{m_{t}}\right\|_{\Gamma_{2}}^{2}+\left\|\sqrt{K} \delta_{m}\right\|_{\Gamma_{2}}^{2}+ \\
+2 \int_{0}^{t}\left\|\sqrt{D} \delta_{m_{t}}\right\|_{\Gamma_{2}}^{2} d s+2 \int_{0}^{t}\left[\left(\left|U_{m_{t}}\right|^{q_{1}+1}, 1\right)_{1}+\left(\left|V_{m_{t}}\right|^{q_{2}+1}, 1\right)_{2}\right] d s \leq \\
\leq\left\|U_{1 m}\right\|_{1}^{2}+\left\|\nabla U_{0 m}\right\|_{1}^{2}+\left\|V_{1 m}\right\|_{2}^{2}+\left\|\nabla V_{0 m}\right\|_{2}^{2}+ \\
+\left\|\sqrt{M}\left(\gamma_{1}\left(U_{0 m}-V_{0 m}\right)+\gamma_{0}\left(\rho\left(U_{1 m}\right)\right)\right)\right\|_{\Gamma_{2}}^{2}+\left\|\sqrt{K} \delta_{0 m}\right\|_{\Gamma_{2}}^{2}+ \\
+\int_{0}^{t}\|F\|_{1}^{2} d s+\int_{0}^{t}\|G\|_{2}^{2} d s+\int_{0}^{t}\left(\left\|U_{m_{t}}\right\|_{1}^{2}+\left\|V_{m_{t}}\right\|_{2}^{2}\right) d s
\end{gathered}
$$

whence by (2.1), (2.4), (3.9)-(3.12) and Gronwall's inequality, we deduce that

$$
\begin{aligned}
& \left\|U_{m_{t}}\right\|_{1}^{2}+\left\|\nabla U_{m}\right\|_{1}^{2}+\left\|V_{m_{t}}\right\|_{2}^{2}+\left\|\nabla V_{m}\right\|_{2}^{2}+\left\|\delta_{m_{t}}\right\|_{\Gamma_{2}}^{2}+\left\|\delta_{m}\right\|_{\Gamma_{2}}^{2}+ \\
& +\int_{0}^{t}\left\|\delta_{m_{t}}\right\|_{\Gamma_{2}}^{2} d s+\int_{0}^{t}\left[\left(\left|U_{m_{t}}\right|^{q_{1}+1}, 1\right)_{1}+\left(\left|V_{m_{t}}\right|^{q_{2}+1}, 1\right)_{2}\right] d s \leq C_{1},
\end{aligned}
$$

where $C_{1}$ is a positive constant, which does not depend on $m$. This is Estimate 1.

Estimate 2. First of all, we estimate $\left\|U_{m_{t t}}(0)\right\|_{1}^{2},\left\|V_{m_{t t}}(0)\right\|_{2}^{2}$ and $\left\|\delta_{m_{t t}}(0)\right\|_{\Gamma_{2}}^{2}$. Taking $\Phi=U_{m_{t t}}$ in (3.21), $\Psi=V_{m_{t t}}$ in (3.22), $e=\delta_{m_{t t}}$ in (3.23) and putting $t=0$, we come to

$$
\begin{gathered}
\left\|U_{m_{t t}}(0)\right\|_{1}^{2}=\left(\Delta U_{0 m}-\left|U_{1 m}\right|^{q_{1}-1} U_{1 m}+F(x, 0), U_{m_{t t}}(0)\right)_{1} \leq \\
\leq\left\|\Delta U_{0 m}-\left|U_{1 m}\right|^{q_{1}-1} U_{1 m}+F(x, 0)\right\|_{1}\left\|U_{m_{t t}}(0)\right\|_{1}, \\
\left\|V_{m_{t t}}(0)\right\|_{2}^{2}=\left(\Delta V_{0 m}-\left|V_{1 m}\right|^{q_{2}-1} V_{1 m}+G(x, 0), V_{m_{t t}}(0)\right)_{2} \leq \\
\leq\left\|\Delta V_{0 m}-\left|V_{1 m}\right|^{q_{2}-1} V_{1 m}+G(x, 0)\right\|_{2}\left\|V_{m_{t t}}(0)\right\|_{2}, \\
\left\|\sqrt{M} \delta_{m_{t t}}(0)\right\|_{\Gamma_{2}}^{2}= \\
=\left(-D\left(\gamma_{1}\left(U_{0 m}-V_{0 m}\right)+\gamma_{0}\left(\rho\left(U_{1 m}\right)\right)\right)-K \delta_{0 m}-\gamma_{0}\left(U_{1 m}\right), \delta_{m_{t t}}(0)\right)_{\Gamma_{2}} \leq \\
\leq\left\|-D\left(\gamma_{1}\left(U_{0 m}-V_{0 m}\right)+\gamma_{0}\left(\rho\left(U_{1 m}\right)\right)\right)-K \delta_{0 m}-\gamma_{0}\left(U_{1 m}\right)\right\|_{\Gamma_{2}}\left\|\delta_{m_{t t}}(0)\right\|_{\Gamma_{2}},
\end{gathered}
$$

hence by $(2.1),(2.4),(3.9)-(3.12)$ we have

$$
\left\|U_{m_{t t}}(0)\right\|_{1}^{2}+\left\|V_{m_{t t}}(0)\right\|_{2}^{2}+\left\|\delta_{m_{t t}}(0)\right\|_{\Gamma_{2}}^{2} \leq C_{2}
$$

where $C_{2}$ is a positive constant, which does not depend on $m$. 
Differentiating (3.21), (3.22), (3.23) and taking $\Phi=2 U_{m_{t t}}, \Psi=2 V_{m_{t t}}, e=$ $2 \delta_{m_{t t}}$, after standard calculations we obtain

$$
\begin{gathered}
\frac{d}{d t}\left(\left\|U_{m_{t t}}\right\|_{1}^{2}+\left\|\nabla U_{m_{t}}\right\|_{1}^{2}+\left\|V_{m_{t t}}\right\|_{2}^{2}+\left\|\nabla V_{m_{t}}\right\|_{2}^{2}+\left\|\sqrt{M} \delta_{m_{t t}}\right\|_{\Gamma_{2}}^{2}+\left\|\sqrt{K} \delta_{m_{t}}\right\|_{\Gamma_{2}}^{2}\right)- \\
-\left(\frac{\partial U_{m_{t}}}{\partial \nu}, \gamma_{0}\left(2 U_{m_{t t}}\right)\right)_{\Gamma_{2}}+\left(\frac{\partial V_{m_{t}}}{\partial \nu}, \gamma_{0}\left(2 V_{m_{t t}}\right)\right)_{\Gamma_{2}}+2\left\|\sqrt{D} \delta_{m_{t t}}\right\|_{\Gamma_{2}}^{2}+ \\
+\left(\gamma_{0}\left(U_{m_{t t}}\right), 2 \delta_{m_{t t}}\right)_{\Gamma_{2}}+2 q_{1} \int_{\Omega_{1}}\left|U_{m_{t}}\right|^{q_{1}-1} U_{m_{t t}}^{2} d x+2 q_{2} \int_{\Omega_{2}}\left|V_{m_{t}}\right|^{q_{2}-1} V_{m_{t t}}^{2} d x= \\
=2\left(F_{t}, U_{m_{t t}}\right)_{1}+2\left(G_{t}, V_{m_{t t}}\right)_{2},
\end{gathered}
$$

whence using (3.20) and Young inequality, we get

$$
\begin{gathered}
\frac{d}{d t}\left(\left\|U_{m_{t t}}\right\|_{1}^{2}+\left\|\nabla U_{m_{t}}\right\|_{1}^{2}+\left\|V_{m_{t t}}\right\|_{2}^{2}+\left\|\nabla V_{m_{t}}\right\|_{2}^{2}+\left\|\sqrt{M} \delta_{m_{t t}}\right\|_{\Gamma_{2}}^{2}+\left\|\sqrt{K} \delta_{m_{t}}\right\|_{\Gamma_{2}}^{2}\right)+ \\
+2\left\|\sqrt{D} \delta_{m_{t t}}\right\|_{\Gamma_{2}}^{2}+\left(\rho^{\prime}\left(U_{m_{t}}\right) U_{m_{t t}}, \gamma_{0}\left(2 U_{m_{t t}}\right)\right)_{\Gamma_{2}}+ \\
+\frac{8 q_{1}}{\left(q_{1}+1\right)^{2}} \int_{\Omega_{1}}\left(\frac{\partial}{\partial t}\left(\left|U_{m_{t}}\right|^{\frac{q_{1}-1}{2}} U_{m_{t}}\right)\right)^{2} d x+ \\
+\frac{8 q_{2}}{\left(q_{2}+1\right)^{2}} \int_{\Omega_{2}}\left(\frac{\partial}{\partial t}\left(\left|V_{m_{t}}\right|^{\frac{q_{2}-1}{2}} V_{m_{t}}\right)\right)^{2} d x \leq\left\|F_{t}\right\|_{1}^{2}+\left\|G_{t}\right\|_{2}^{2}+\left\|U_{m_{t t}}\right\|_{1}^{2}+\left\|V_{m_{t t}}\right\|_{2}^{2}
\end{gathered}
$$

integrating this over $(0, t)$ and using the fact that by $(2.5): \rho^{\prime}(s) \geq 0, \forall s \in$ $(-\infty,+\infty)$, we have

$$
\begin{gathered}
\left\|U_{m_{t t}}\right\|_{1}^{2}+\left\|\nabla U_{m_{t}}\right\|_{1}^{2}+\left\|V_{m_{t t}}\right\|_{2}^{2}+\left\|\nabla V_{m_{t}}\right\|_{2}^{2}+\left\|\sqrt{M} \delta_{m_{t t}}\right\|_{\Gamma_{2}}^{2}+\left\|\sqrt{K} \delta_{m_{t}}\right\|_{\Gamma_{2}}^{2}+ \\
+2 \int_{0}^{t}\left\|\sqrt{D} \delta_{m_{t t}}\right\|_{\Gamma_{2}}^{2} d s+\frac{8 q_{1}}{\left(q_{1}+1\right)^{2}} \int_{0}^{t} \int_{\Omega_{1}}\left(\frac{\partial}{\partial t}\left(\left|U_{m_{t}}\right|^{\frac{q_{1}-1}{2}} U_{m_{t}}\right)\right)^{2} d x d s+ \\
+\frac{8 q_{2}}{\left(q_{2}+1\right)^{2}} \int_{0}^{t} \int_{\Omega_{2}}\left(\frac{\partial}{\partial t}\left(\left|V_{m_{t}}\right|^{\frac{q_{2}-1}{2}} V_{m_{t}}\right)\right)^{2} d x d s \leq \\
\leq\left\|U_{m_{t t}}(0)\right\|_{1}^{2}+\left\|\nabla U_{1_{m}}\right\|_{1}^{2}+\left\|V_{m_{t t}}(0)\right\|_{2}^{2}+\left\|\nabla V_{1_{m}}\right\|_{2}^{2}+\left\|\sqrt{M} \delta_{m_{t t}}(0)\right\|_{\Gamma_{2}}^{2}+ \\
+\left\|\sqrt{K}\left(\gamma_{1}\left(U_{0_{m}}-V_{0_{m}}\right)+\gamma_{0}\left(\rho\left(U_{1_{m}}\right)\right)\right)\right\|_{\Gamma_{2}}^{2}+ \\
+\int_{0}^{t}\left\|F_{t}\right\|_{1}^{2} d s+\int_{0}^{t}\left\|G_{t}\right\|_{2}^{2} d s+\int_{0}^{t}\left(\left\|U_{m_{t t}}\right\|_{1}^{2}+\left\|V_{m_{t t}}\right\|_{2}^{2}\right) d s .
\end{gathered}
$$

Using (3.9)-(3.12), (3.24) and Gronwall's inequality we can obtain

$$
\begin{gathered}
\left\|U_{m_{t t}}\right\|_{1}^{2}+\left\|\nabla U_{m_{t}}\right\|_{1}^{2}+\left\|V_{m_{t t}}\right\|_{2}^{2}+\left\|\nabla V_{m_{t}}\right\|_{2}^{2}+\left\|\sqrt{M} \delta_{m_{t t}}\right\|_{\Gamma_{2}}^{2}+\left\|\sqrt{K} \delta_{m_{t}}\right\|_{\Gamma_{2}}^{2}+ \\
+2 \int_{0}^{t}\left\|\sqrt{D} \delta_{m_{t t}}\right\|_{\Gamma_{2}}^{2} d s+\frac{8 q_{1}}{\left(q_{1}+1\right)^{2}} \int_{0}^{t} \int_{\Omega_{1}}\left(\frac{\partial}{\partial t}\left(\left|U_{m_{t}}\right|^{\frac{q_{1}-1}{2}} U_{m_{t}}\right)\right)^{2} d x d s+
\end{gathered}
$$




$$
+\frac{8 q_{2}}{\left(q_{2}+1\right)^{2}} \int_{0}^{t} \int_{\Omega_{2}}\left(\frac{\partial}{\partial t}\left(\left|V_{m_{t}}\right|^{\frac{q_{2}-1}{2}} V_{m_{t}}\right)\right)^{2} d x d s \leq C_{3},
$$

where $C_{3}$ is a positive constant, which does not depend on $m$. This is Estimate 2 .

Passage to the limit. Using the estimates 1,2 and compactness argument, we can see that there exist a subsequence of $\left\{U_{m}\right\}$, a subsequence of $\left\{V_{m}\right\}$ and a subsequence of $\left\{\delta_{m}\right\}$ which will be denoted by the same notations, and the functions $U, V$ and $\delta$, such that

$$
\begin{aligned}
U_{m} & \rightarrow U \text { weakly star in } L^{\infty}\left(0, T ; H_{\Gamma_{1}}^{1}\left(\Omega_{1}\right)\right), \\
V_{m} & \rightarrow V \text { weakly star in } L^{\infty}\left(0, T ; H^{1}\left(\Omega_{2}\right)\right) ; \\
U_{m_{t}} & \rightarrow U_{t} \text { weakly star in } L^{\infty}\left(0, T ; H_{\Gamma_{1}}^{1}\left(\Omega_{1}\right)\right), \\
V_{m_{t}} & \rightarrow V_{t} \text { weakly star in } L^{\infty}\left(0, T ; H^{1}\left(\Omega_{2}\right)\right) ; \\
U_{m_{t t}} & \rightarrow U_{t t} \text { weakly star in } L^{\infty}\left(0, T ; L^{2}\left(\Omega_{1}\right)\right), \\
V_{m_{t t}} & \rightarrow V_{t t} \text { weakly star in } L^{\infty}\left(0, T ; L^{2}\left(\Omega_{2}\right)\right) ; \\
\delta_{m} & \rightarrow \delta \text { weakly star in } L^{\infty}\left(0, T ; L^{2}\left(\Gamma_{2}\right)\right), \\
\delta_{m_{t}} & \rightarrow \delta_{t} \text { weakly star in } L^{\infty}\left(0, T ; L^{2}\left(\Gamma_{2}\right)\right) ; \\
\delta_{m_{t t}} & \rightarrow \delta_{t t} \text { weakly star in } L^{\infty}\left(0, T ; L^{2}\left(\Gamma_{2}\right)\right) .
\end{aligned}
$$

From [27, Theorem 3.1] we obtain that

$$
\begin{aligned}
U_{m} & \rightarrow U \text { strongly in } C\left([0, T] ; H_{\Gamma_{1}}^{1}\left(\Omega_{1}\right)\right), \\
U_{m_{t}} & \rightarrow U_{t} \text { strongly in } C\left([0, T] ; L^{2}\left(\Omega_{1}\right)\right), \\
V_{m} & \rightarrow V \text { strongly in } C\left([0, T] ; H^{1}\left(\Omega_{2}\right)\right), \\
V_{m_{t}} & \rightarrow V_{t} \text { strongly in } C\left([0, T] ; L^{2}\left(\Omega_{2}\right)\right),
\end{aligned}
$$

and consequently:

$$
U_{m} \rightarrow U, U_{m_{t}} \rightarrow U_{t} \text { a.e. in } \Omega_{1} \times(0, T), V_{m} \rightarrow V, V_{m_{t}} \rightarrow V_{t} \text { a.e. in } \Omega_{2} \times(0, T) \text {; }
$$

therefore

$$
\begin{gathered}
\left|U_{m_{t}}\right|^{q_{1}-1} U_{m_{t}} \rightarrow\left|U_{t}\right|^{q_{1}-1} U_{t}, \text { a.e. in } \Omega_{1} \times(0, T), \\
\left|V_{m_{t}}\right|^{q_{2}-1} V_{m_{t}} \rightarrow\left|V_{t}\right|^{q_{2}-1} V_{t}, \text { a.e. in } \Omega_{2} \times(0, T) .
\end{gathered}
$$

Since by Estimate 1:

$$
U_{m_{t}} \in L^{q_{1}+1}\left(\Omega_{1} \times(0, T)\right), \quad V_{m_{t}} \in L^{q_{2}+1}\left(\Omega_{2} \times(0, T)\right),
$$

we obtain that

$$
\begin{gathered}
\left|U_{m_{t}}\right|^{q_{1}-1} U_{m_{t}} \in\left(L^{q_{1}+1}\left(\Omega_{1} \times(0, T)\right)\right)^{\prime}=L^{\frac{q_{1}+1}{q_{1}}}\left(\Omega_{1} \times(0, T)\right), \\
\left|V_{m_{t}}\right|^{q_{2}-1} V_{m_{t}} \in\left(L^{q_{2}+1}\left(\Omega_{2} \times(0, T)\right)\right)^{\prime}=L^{\frac{q_{2}+1}{q_{2}}}\left(\Omega_{2} \times(0, T)\right) .
\end{gathered}
$$

Therefore using estimate 1 we can see that there exist a subsequence of $\left\{U_{m}\right\}$ and a subsequence of $\left\{V_{m}\right\}$, which we still denote by the same notations, and the functions $\psi, \chi$ such that as $m \rightarrow \infty$

$$
\begin{gathered}
\left|U_{m_{t}}\right|^{q_{1}-1} U_{m_{t}} \rightarrow \psi \text { weakly star in } L^{\frac{q_{1}+1}{q_{1}}}\left(\Omega_{1} \times(0, T)\right), \\
\left|V_{m_{t}}\right|^{q_{2}-1} V_{m_{t}} \rightarrow \chi \text { weakly star in } L^{\frac{q_{2}+1}{q_{2}}}\left(\Omega_{2} \times(0, T)\right),
\end{gathered}
$$


whence using (3.30) and [26, Lemma 1.3] we get

$$
\psi=\left|U_{t}\right|^{q_{1}-1} U_{t}, \quad \chi=\left|V_{t}\right|^{q_{2}-1} V_{t}
$$

or

$$
\begin{gathered}
\left|U_{m_{t}}\right|^{q_{1}-1} U_{m_{t}} \rightarrow\left|U_{t}\right|^{q_{1}-1} U_{t} \text { weakly star in } L^{\frac{q_{1}+1}{q_{1}}}\left(\Omega_{1} \times(0, T)\right), \\
\left|V_{m_{t}}\right|^{q_{2}-1} V_{m_{t}} \rightarrow\left|V_{t}\right|^{q_{2}-1} V_{t} \text { weakly star in } L^{\frac{q_{2}+1}{q_{2}}}\left(\Omega_{2} \times(0, T)\right) .
\end{gathered}
$$

Taking into account the convergences in (3.25)-(3.29), (3.31), (3.32) we can pass to the limit in (3.21)-(3.23) and (3.20) as $m \rightarrow \infty$ :

$$
\begin{gathered}
\left(U_{t t}, \Phi\right)_{1}+(\nabla U, \nabla \Phi)_{1}- \\
-\left(\frac{\partial U}{\partial \nu}, \gamma_{0}(\Phi)\right)_{\Gamma_{2}}+\left(\left|U_{t}\right|^{q_{1}-1} U_{t}, \Phi\right)_{1}=(F, \Phi)_{1}, \\
\left(V_{t t}, \Psi\right)_{2}+(\nabla V, \nabla \Psi)_{2}+ \\
+\left(\frac{\partial V}{\partial \nu}, \gamma_{0}(\Psi)\right)_{\Gamma_{2}}+\left(\left|V_{t}\right|^{q_{2}-1} V_{t}, \Psi\right)_{2}=(G, \Psi)_{2}, \\
\left(M \delta_{t t}+D \delta_{t}+K \delta, e\right)_{\Gamma_{2}}=-\left(\gamma_{0}\left(U_{t}\right), e\right)_{\Gamma_{2}}
\end{gathered}
$$

for all $(\Phi, \Psi, e) \in W$ a.e. in $(0, T)$ and

$$
U=V, \delta_{t}=\frac{\partial U}{\partial \nu}-\frac{\partial V}{\partial \nu}+\rho\left(U_{t}\right), x \in \Gamma_{2}
$$

From (3.33), (3.34) we obtain

$$
\begin{gathered}
\int_{\Omega_{1}} U_{t t} \Phi d x-(\Delta U, \Phi)_{D^{\prime}\left(\Omega_{1}\right) \times D\left(\Omega_{1}\right)}+\int_{\Omega_{1}}\left|U_{t}\right|^{q_{1}-1} U_{t} \Phi d x=\int_{\Omega_{1}} F \Phi d x, \\
\int_{\Omega_{2}} V_{t t} \Psi d x-(\Delta V, \Psi)_{D^{\prime}\left(\Omega_{2}\right) \times D\left(\Omega_{2}\right)}+\int_{\Omega_{2}}\left|V_{t}\right|^{q_{2}-1} V_{t} \Psi d x=\int_{\Omega_{2}} G \Psi d x
\end{gathered}
$$

for all $\Phi \in D\left(\Omega_{1}\right), \Psi \in D\left(\Omega_{2}\right)$ a. e. in $(0, T)$. Therefore $\left(-\Delta U+\left|U_{t}\right|^{q_{1}-1} U_{t}\right)(t) \in$ $L^{2}\left(\Omega_{1}\right),\left(-\Delta V+\left|V_{t}\right|^{q_{2}-1} V_{t}\right)(t) \in L^{2}\left(\Omega_{2}\right)$ a.e. on $(0, T)$ and

$$
\begin{gathered}
U_{t t}-\Delta U+\left|U_{t}\right|^{q_{1}-1} U_{t}=F \text { a.e. in } \Omega_{1} \times(0, T), \\
V_{t t}-\Delta V+\left|V_{t}\right|^{q_{2}-1} V_{t}=G \text { a.e. in } \Omega_{2} \times(0, T) .
\end{gathered}
$$

From (3.35) we can see that $(U, V, \delta)$ satisfies the boundary condition (3.3).

The initial conditions (3.6)-(3.8) can be proved in a standard way and this completes the proof of the existence of solutions.

Uniqueness. The uniqueness of solution to the problem (3.1)-(3.8) is obtained by energy method as follows.

Let $\left(U_{1}, V_{1}, \delta_{1}\right)$ and $\left(U_{2}, V_{2}, \delta_{2}\right)$ be two solutions of the problem (3.1)-(3.8). We have that $U_{1}-U_{2}=\widetilde{U}, V_{1}-V_{2}=\widetilde{V}, \delta_{1}-\delta_{2}=\widetilde{\delta}$ satisfy

$$
\begin{gathered}
\left(\widetilde{U}_{t t}, \Phi\right)_{1}+(\nabla \widetilde{U}, \nabla \Phi)_{1}- \\
-\left(\frac{\partial \widetilde{U}}{\partial \nu}, \gamma_{0}(\Phi)\right)_{\Gamma_{2}}+\int_{\Omega_{1}}\left(\left|U_{1 t}\right|^{q_{1}-1} U_{1 t}-\left|U_{2 t}\right|^{q_{1}-1} U_{2 t}\right) \Phi d x=0,
\end{gathered}
$$




$$
\begin{gathered}
\left(\widetilde{V}_{t t}, \Psi\right)_{2}+(\nabla \widetilde{V}, \nabla \Psi)_{2}+ \\
+\left(\frac{\partial \widetilde{V}}{\partial \nu}, \gamma_{0}(\Psi)\right)_{\Gamma_{2}}+\int_{\Omega_{2}}\left(\left|V_{1 t}\right|^{q_{2}-1} V_{1 t}-\left|V_{2 t}\right|^{q_{2}-1} V_{2 t}\right) \Psi d x=0 \\
\left(M \widetilde{\delta}_{t t}+D \widetilde{\delta}_{t}+K \widetilde{\delta}, e\right)_{\Gamma_{2}}=-\left(\gamma_{0}\left(\widetilde{U}_{t}\right), e\right)_{\Gamma_{2}}
\end{gathered}
$$

for all $(\Phi, \Psi, e) \in W$ a. e. in $(0, T)$;

$$
\begin{gathered}
\widetilde{U}=0 \text { a.e. on } \Gamma_{1} \times(0, T), \\
\widetilde{U}=\widetilde{V}, \frac{\partial \widetilde{U}}{\partial \nu}-\frac{\partial \widetilde{V}}{\partial \nu}+\rho\left(U_{1 t}\right)-\rho\left(U_{2 t}\right)=\widetilde{\delta}_{t} \text { a.e. on } \Gamma_{2} \times(0, T), \\
\widetilde{U}(x, 0)=0, \widetilde{U}_{t}(x, 0)=0, x \in \Omega_{1}, \\
\widetilde{V}(x, 0)=0, \widetilde{V}_{t}(x, 0)=0, x \in \Omega_{2}, \\
\widetilde{\delta}(x, 0)=0, \widetilde{\delta}_{t}(x, 0)=0, x \in \Gamma_{2},
\end{gathered}
$$

Taking $\Phi=2 \widetilde{U}_{t}$ in (3.36), $\Psi=2 \widetilde{V}_{t}$ in (3.37), $e=2 \widetilde{\delta}_{t}$ in (3.38) and using (3.39), (3.40), we get

$$
\begin{aligned}
\frac{d}{d t}\left(\left\|\widetilde{U}_{t}\right\|_{1}^{2}\right. & \left.+\|\nabla \widetilde{U}\|_{1}^{2}+\left\|\widetilde{V}_{t}\right\|_{2}^{2}+\|\nabla \widetilde{V}\|_{2}^{2}+\left\|\sqrt{M} \widetilde{\delta}_{t}\right\|_{\Gamma_{2}}^{2}+\|\sqrt{K} \widetilde{\delta}\|_{\Gamma_{2}}^{2}\right)+ \\
+ & 2\left(\rho\left(U_{1 t}\right)-\rho\left(U_{2 t}\right), U_{1 t}-U_{2 t}\right)_{\Gamma_{2}}+2\left\|\sqrt{D} \widetilde{\delta}_{t}\right\|_{\Gamma_{2}}^{2}+ \\
& +2\left(\left|U_{1 t}\right|^{q_{1}-1} U_{1 t}-\left|U_{2 t}\right|^{q_{1}-1} U_{2 t}, U_{1 t}-U_{2 t}\right)_{1}+ \\
& +2\left(\left|V_{1 t}\right|^{q_{2}-1} V_{1 t}-\left|V_{2 t}\right|^{q_{2}-1} V_{2 t}, V_{1 t}-V_{2 t}\right)_{2}=0
\end{aligned}
$$

Since

$$
\begin{gathered}
\left(\left|U_{1 t}\right|^{q_{1}-1} U_{1 t}-\left|U_{2 t}\right|^{q_{1}-1} U_{2 t}, U_{1 t}-U_{2 t}\right)_{1} \geq 0, \\
\left(\left|V_{1 t}\right|^{q_{2}-1} V_{1 t}-\left|V_{2 t}\right|^{q_{2}-1} V_{2 t}, V_{1 t}-V_{2 t}\right)_{2} \geq 0
\end{gathered}
$$

and by $(2.5)$ :

$$
\left(\rho\left(U_{1 t}\right)-\rho\left(U_{2 t}\right), U_{1 t}-U_{2 t}\right)_{\Gamma_{2}} \geq 0
$$

then using (3.41)-(3.43) in last equality, we obtain

$$
\begin{gathered}
\left\|\widetilde{U}_{t}\right\|_{1}^{2}+\|\nabla \widetilde{U}\|_{1}^{2}+\left\|\widetilde{V}_{t}\right\|_{2}^{2}+\|\nabla \widetilde{V}\|_{2}^{2}+ \\
+\left\|\sqrt{M} \widetilde{\delta}_{t}\right\|_{\Gamma_{2}}^{2}+\|\sqrt{K} \widetilde{\delta}\|_{\Gamma_{2}}^{2}+2 \int_{0}^{t}\left\|\sqrt{D} \widetilde{\delta}_{t}\right\|_{\Gamma_{2}}^{2} d s \leq 0 .
\end{gathered}
$$

This inequality yields $\widetilde{U}=0, \widetilde{V}=0, \widetilde{\delta}=0$. Lemma 3.1 is proved.

For

$$
\begin{gathered}
u \in C\left([0, T] ; H_{\Gamma_{1}}^{1}\left(\Omega_{1}\right)\right) \cap C^{1}\left([0, T] ; L^{2}\left(\Omega_{1}\right)\right), \\
v \in C\left([0, T] ; H^{1}\left(\Omega_{2}\right)\right) \cap C^{1}\left([0, T] ; L^{2}\left(\Omega_{2}\right)\right)
\end{gathered}
$$


$\left(\left.u\right|_{\Gamma_{2}}=\left.v\right|_{\Gamma_{2}}\right)$ given we consider the following problem:

$$
\left\{\begin{array}{c}
U_{t t}-\Delta U+\left|U_{t}\right|^{q_{1}-1} U_{t}=f(u) \text { in } \Omega_{1} \times(0, T), \\
V_{t t}-\Delta V+\left|V_{t}\right|^{q_{2}-1} V_{t}=g(v) \text { in } \Omega_{2} \times(0, T), \\
M \delta_{t t}+D \delta_{t}+K \delta=-U_{t} \text { on } \Gamma_{2} \times(0, T), \\
U=0 \text { on } \Gamma_{1} \times(0, T), \\
U=V, \frac{\partial U}{\partial \nu}-\frac{\partial V}{\partial \nu}+\rho\left(U_{t}\right)=\delta_{t} \text { on } \Gamma_{2}, \\
U(x, 0)=u_{0}(x), U_{t}(x, 0)=u_{1}(x), x \in \Omega_{1}, \\
V(x, 0)=v_{0}(x), V_{t}(x, 0)=v_{1}(x), x \in \Omega_{2}, \\
\delta(x, 0)=\delta_{0}(x), \delta_{t}(x, 0)=\delta_{1}(x), x \in \Gamma_{2} .
\end{array}\right.
$$

Lemma 3.2. Suppose that (2.1)-(2.5) hold and let $u_{0} \in H_{\Gamma_{1}}^{1}\left(\Omega_{1}\right), u_{1} \in L^{2 q_{1}}\left(\Omega_{1}\right)$, $v_{0} \in H^{1}\left(\Omega_{2}\right), v_{1} \in L^{2 q_{2}}\left(\Omega_{2}\right), \delta_{0} \in L^{2}\left(\Gamma_{2}\right), \delta_{1} \in L^{2}\left(\Gamma_{2}\right)$. Then, there exist $T>0$ and a unique solution $(U, V, \delta)$ to the problem (3.44) such that

$$
\begin{gathered}
U \in C\left([0, T] ; H_{\Gamma_{1}}^{1}\left(\Omega_{1}\right)\right), U_{t} \in C\left([0, T] ; L^{2}\left(\Omega_{1}\right)\right) \cap L^{q_{1}+1}\left(\Omega_{1} \times(0, T)\right), \\
V \in C\left([0, T] ; H^{1}\left(\Omega_{2}\right)\right), V_{t} \in C\left([0, T] ; L^{2}\left(\Omega_{2}\right)\right) \cap L^{q_{2}+1}\left(\Omega_{2} \times(0, T)\right), \\
\delta, \delta_{t} \in L^{\infty}\left(0, T ; L^{2}\left(\Gamma_{2}\right)\right) .
\end{gathered}
$$

Proof of Lemma 3.2. By the same methods as in [21], we approximate $u \in$ $C\left([0, T] ; H_{\Gamma_{1}}^{1}\left(\Omega_{1}\right)\right) \cap C^{1}\left([0, T] ; L^{2}\left(\Omega_{1}\right)\right), v \in C\left([0, T] ; H^{1}\left(\Omega_{2}\right)\right) \cap C^{1}\left([0, T] ; L^{2}\left(\Omega_{2}\right)\right)$ by sequences $\left\{u_{\mu}\right\}_{\mu \in N}$ in $C\left([0, T] ; C_{0}^{\infty}\left(\Omega_{1}\right)\right),\left\{v_{\mu}\right\}_{\mu \in N}$ in $C\left([0, T] ; C^{\infty}\left(\Omega_{2}\right)\right)$ by standard convolution argument as in [11]. Next we approximate the initial data $u_{0} \in H_{\Gamma_{1}}^{1}\left(\Omega_{1}\right)$ by a sequence $\left\{u_{\mu}^{0}\right\}_{\mu \in N}$ in $H_{\Gamma_{1}}^{1}\left(\Omega_{1}\right) \cap H^{2}\left(\Omega_{1}\right)$, the initial data $v_{0} \in H^{1}\left(\Omega_{2}\right)$ by a sequence $\left\{v_{\mu}^{0}\right\}_{\mu \in N}$ in $H^{2}\left(\Omega_{2}\right)$, the initial data $u_{1} \in L^{2 q_{1}}\left(\Omega_{1}\right)$ by a sequence $\left\{u_{\mu}^{1}\right\}_{\mu \in N}$ in $C^{\infty}\left(\Omega_{1}\right)$, the initial data $v_{1} \in L^{2 q_{2}}\left(\Omega_{2}\right)$ by a sequence $\left\{v_{\mu}^{1}\right\}_{\mu \in N}$ in $C^{\infty}\left(\Omega_{2}\right), \delta_{0} \in L^{2}\left(\Gamma_{2}\right)$ by a sequence $\left\{\delta_{\mu}^{0}\right\}_{\mu \in N}$ in $C^{\infty}\left(\Gamma_{2}\right)$ and $\delta_{1} \in$ $L^{2}\left(\Gamma_{2}\right)$ by a sequence $\left\{\delta_{\mu}^{1}\right\}_{\mu \in N}$ in $C^{\infty}\left(\Gamma_{2}\right)$. Then we consider the set of following problems

$$
\left\{\begin{array}{c}
U_{\mu_{t t}}-\Delta U_{\mu}+\left|U_{\mu_{t}}\right|^{q_{1}-1} U_{\mu_{t}}=f\left(u_{\mu}\right) \text { in } \Omega_{1} \times(0, T), \\
V_{\mu_{t t}}-\Delta V_{\mu}+\left|V_{\mu_{t}}\right|^{q_{2}-1} V_{\mu_{t}}=g\left(v_{\mu}\right) \text { in } \Omega_{2} \times(0, T), \\
M \delta_{\mu_{t t}}+D \delta_{\mu_{t}}+K \delta_{\mu}=-U_{\mu_{t}} \text { on } \Gamma_{2} \times(0, T), \\
U_{\mu}=0 \text { on } \Gamma_{1} \times(0, T), \\
U_{\mu}=V_{\mu}, \frac{\partial U_{\mu}}{\partial \nu}-\frac{\partial V_{\mu}}{\partial \nu}+\rho\left(U_{\mu_{t}}\right)=\delta_{\mu_{t}} \text { on } \Gamma_{2}, \\
U_{\mu}(x, 0)=u_{\mu}^{0}(x), U_{\mu_{t}}(x, 0)=u_{\mu}^{1}(x), x \in \Omega_{1}, \\
V_{\mu}(x, 0)=v_{\mu}^{0}(x), V_{\mu_{t}}(x, 0)=v_{\mu}^{1}(x), x \in \Omega_{2}, \\
\delta_{\mu}(x, 0)=\delta_{\mu}^{0}(x), \delta_{\mu_{t}}(x, 0)=\delta_{\mu}^{1}(x), x \in \Gamma_{2} .
\end{array}\right.
$$

By (2.2)-(2.3) we obtain $f\left(u_{\mu}\right) \in H^{1}\left(0, T ; L^{2}\left(\Omega_{1}\right)\right), g\left(v_{\mu}\right) \in H^{1}\left(0, T ; L^{2}\left(\Omega_{2}\right)\right)$. Consequently, Lemma 3.1 guarantees the existence of a sequence of unique solutions $\left(U_{\mu}, V_{\mu}, \delta_{\mu}\right)$ to the problem (3.45) satisfying (3.13)-(3.16). Our goal now is to show that the sequence of solutions $\left(U_{\mu}, V_{\mu}, \delta_{\mu}\right)$ converges to the solution $(U, V, \delta)$ of (3.44); it is suffices to show that $\left(U_{\mu}, V_{\mu}, \delta_{\mu}\right)$ is a Cauchy sequence in the space

$$
\begin{gathered}
Z_{T}=\left\{(U, V, \delta): U \in C\left([0, T] ; H_{\Gamma_{1}}^{1}\left(\Omega_{1}\right)\right) \cap C^{1}\left([0, T] ; L^{2}\left(\Omega_{1}\right)\right),\right. \\
V \in C\left([0, T] ; H^{1}\left(\Omega_{2}\right)\right) \cap C^{1}\left([0, T] ; L^{2}\left(\Omega_{2}\right)\right), \delta \in L^{\infty}\left(0, T ; L^{2}\left(\Gamma_{2}\right)\right), \\
\left.\delta_{t} \in L^{\infty}\left(0, T ; L^{2}\left(\Gamma_{2}\right)\right)\right\}
\end{gathered}
$$


endowed with the norm

$$
\begin{gathered}
\|(U, V, \delta)\|_{Z_{T}}^{2}= \\
=\max _{0 \leq t \leq T}\left(\left\|U_{t}\right\|_{1}^{2}+\|\nabla U\|_{1}^{2}+\left\|V_{t}\right\|_{2}^{2}+\|\nabla V\|_{2}^{2}+\left\|\delta_{t}\right\|_{\Gamma_{2}}^{2}+\|\delta\|_{\Gamma_{2}}^{2}\right) .
\end{gathered}
$$

We set $\tilde{u}=u_{\mu}-u_{\tau}, \tilde{v}=v_{\mu}-v_{\tau}, \tilde{U}=U_{\mu}-U_{\tau}, \tilde{V}=V_{\mu}-V_{\tau}, \tilde{\delta}=\delta_{\mu}-\delta_{\tau}$. It is easy to see that $(\tilde{U}, \tilde{V}, \tilde{\delta})$ satisfies

$$
\left\{\begin{array}{l}
\widetilde{U}_{t t}-\Delta \widetilde{U}+\left|U_{\mu_{t}}\right|^{q_{1}-1} U_{\mu_{t}}-\left|U_{\tau_{t}}\right|^{q_{1}-1} U_{\tau_{t}}=f\left(u_{\mu}\right)-f\left(u_{\tau}\right) \text { in } \Omega_{1} \times(0, T), \\
\widetilde{V}_{t t}-\Delta \widetilde{V}+\left|V_{\mu_{t}}\right|^{q_{2}-1} V_{\mu_{t}}-\left|V_{\tau_{t}}\right|^{q_{2}-1} V_{\tau_{t}}=g\left(v_{\mu}\right)-g\left(v_{\tau}\right) \text { in } \Omega_{2} \times(0, T), \\
M \widetilde{\delta}_{t t}+D \widetilde{\delta}_{t}+K \widetilde{\delta}=-\widetilde{U}_{t} \text { on } \Gamma_{2} \times(0, T) \\
\widetilde{U}=0 \text { on } \Gamma_{1} \times(0, T) \\
\widetilde{U}=\widetilde{V}, \frac{\partial \widetilde{U}}{\partial \nu}-\frac{\partial \widetilde{V}}{\partial \nu}+\rho\left(U_{\mu_{t}}\right)-\rho\left(U_{\tau_{t}}\right)=\widetilde{\delta}_{t} \text { on } \Gamma_{2}, \\
\widetilde{U}(x, 0)=\widetilde{U}_{0}(x)=u_{\mu}^{0}(x)-u_{\tau}^{0}(x), \\
\widetilde{U}{ }_{t}(x, 0)=\widetilde{U}_{1}(x)=u_{\mu}^{1}(x)-u_{\tau}^{1}(x), x \in \Omega_{1} \\
\widetilde{V}(x, 0)=\widetilde{V}_{0}(x)=v_{\mu}^{0}(x)-v_{\tau}^{0}(x), \\
\widetilde{V}_{t}(x, 0)=\widetilde{V}_{1}(x)=v_{\mu}^{1}(x)-v_{\tau}^{1}(x), x \in \Omega_{2} \\
\widetilde{\delta}(x, 0)=\widetilde{\delta}_{0}(x)=\delta_{\mu}^{0}(x)-\delta_{\tau}^{0}(x), \\
\widetilde{\delta}_{t}(x, 0)=\widetilde{\delta}_{1}(x)=\delta_{\mu}^{1}(x)-\delta_{\tau}^{1}(x), x \in \Gamma_{2} .
\end{array}\right.
$$

Multiplying the first equation of (3.46) by $2 \widetilde{U}_{t}$, the second equation by $2 \widetilde{V}_{t}$ and the third equation by $2 \widetilde{\delta}_{t}$, integrating over $\Omega_{1} \times(0, T), \Omega_{2} \times(0, T)$ and $\Gamma_{2} \times(0, T)$, respectively, then using the fifth condition in (3.46) and the fact that

$$
\begin{gathered}
\left(\left|U_{\mu_{t}}\right|^{q_{1}-1} U_{\mu_{t}}-\left|U_{\tau_{t}}\right|^{q_{1}-1} U_{\tau_{t}}, U_{\mu_{t}}-U_{\tau_{t}}\right)_{1} \geq 0, \\
\left(\left|V_{\mu_{t}}\right|^{q_{2}-1} V_{\mu_{t}}-\left|V_{\tau_{t}}\right|^{q_{2}-1} V_{\tau_{t}}, V_{\mu_{t}}-V_{\tau_{t}}\right)_{2} \geq 0,
\end{gathered}
$$

we get

$$
\begin{gathered}
\frac{d}{d t}\left(\left\|\widetilde{U}_{t}\right\|_{1}^{2}+\|\nabla \widetilde{U}\|_{1}^{2}+\left\|\widetilde{V}_{t}\right\|_{2}^{2}+\|\nabla \widetilde{V}\|_{2}^{2}+\left\|\sqrt{M} \widetilde{\delta}_{t}\right\|_{\Gamma_{2}}^{2}+\|\sqrt{K} \widetilde{\delta}\|_{\Gamma_{2}}^{2}\right)+ \\
+2\left(\rho\left(U_{\mu_{t}}\right)-\rho\left(U_{\tau_{t}}\right), U_{\mu_{t}}-U_{\tau_{t}}\right)_{\Gamma_{2}}+2\left\|\sqrt{D} \widetilde{\delta}_{t}\right\|_{\Gamma_{2}}^{2} \leq \\
\leq 2\left(f\left(u_{\mu}\right)-f\left(u_{\tau}\right), \widetilde{U}_{t}\right)_{1}+2\left(g\left(v_{\mu}\right)-g\left(v_{\tau}\right), \widetilde{V}_{t}\right)_{2} .
\end{gathered}
$$

Using Hölder's inequality (with $\frac{1}{n}+\frac{n-2}{2 n}+\frac{1}{2}=1$ ) and (2.2) we estimate the right hand side of (3.47) as follows:

$$
\begin{aligned}
\int_{\Omega_{1}}\left(f\left(u_{\mu}\right)-\right. & \left.f\left(u_{\tau}\right)\right) \widetilde{U}_{t} d x \leq \int_{\Omega_{1}} f^{\prime}\left(\theta u_{\mu}+(1-\theta) u_{\tau}\right)|\tilde{u}|\left|\widetilde{U}_{t}\right| d x \leq \\
\leq & c_{2} \int_{\Omega_{1}} \sup \left(\left|u_{\mu}\right|^{p-1},\left|u_{\tau}\right|^{p-1}\right)|\tilde{u}|\left|\widetilde{U}_{t}\right| d x \leq \\
\leq & c_{2}\left(\left\|u_{\mu}\right\|_{L^{(p-1) n}\left(\Omega_{1}\right)}^{p-1}+\left\|u_{\tau}\right\|_{L^{(p-1) n}\left(\Omega_{1}\right)}^{p-1}\right) \times \\
& \times\|\tilde{u}\|_{L^{2 n /(n-2)}\left(\Omega_{1}\right)}\left\|\widetilde{U}_{t}\right\|_{1}(0<\theta<1) ;
\end{aligned}
$$


by a similar way:

$$
\begin{gathered}
\int_{\Omega_{2}}\left(g\left(v_{\mu}\right)-g\left(v_{\tau}\right)\right) \widetilde{V}_{t} d x \leq \\
\leq c_{4}\left(\left\|v_{\mu}\right\|_{L^{(p-1) n}\left(\Omega_{2}\right)}^{p-1}+\left\|v_{\tau}\right\|_{L^{(p-1) n}\left(\Omega_{2}\right)}^{p-1}\right)\|\tilde{v}\|_{L^{2 n /(n-2)}\left(\Omega_{2}\right)}\left\|\widetilde{V}_{t}\right\|_{2} .
\end{gathered}
$$

By the Sobolev embedding $H_{\Gamma_{1}}^{1}\left(\Omega_{1}\right) \mapsto L^{2 n /(n-2)}\left(\Omega_{1}\right)$, Phriedrich's inequality and the condition that $\left.\tilde{u}\right|_{\Gamma_{2}}=\left.\tilde{v}\right|_{\Gamma_{2}}$ we can obtain

$$
\|\tilde{u}\|_{L^{2 n /(n-2)\left(\Omega_{1}\right)}}+\|\tilde{v}\|_{L^{2 n /(n-2)}\left(\Omega_{2}\right)} \leq C_{4}\left(\|\nabla \tilde{u}\|_{1}+\|\nabla \tilde{v}\|_{2}\right) ;
$$

and in a similar way, by (2.3) we have

$$
\begin{gathered}
\left\|u_{\mu}\right\|_{L^{(p-1) n}\left(\Omega_{1}\right)}^{p-1}+\left\|u_{\tau}\right\|_{L^{(p-1) n}\left(\Omega_{1}\right)}^{p-1}+\left\|v_{\mu}\right\|_{L^{(p-1) n}\left(\Omega_{2}\right)}^{p-1}+\left\|v_{\tau}\right\|_{L^{(p-1) n}\left(\Omega_{2}\right)}^{p-1} \leq \\
\leq C_{5}\left(\left\|\nabla u_{\mu}\right\|_{1}^{p-1}+\left\|\nabla u_{\tau}\right\|_{1}^{p-1}+\left\|\nabla v_{\mu}\right\|_{2}^{p-1}+\left\|\nabla v_{\tau}\right\|_{2}^{p-1}\right),
\end{gathered}
$$

where $C_{4}, C_{5}$ are positive constants depending only on $\Omega_{1}, \Omega_{2}$ and $p$. By these inequalities from (3.48), (3.49) we have

$$
\begin{gathered}
\left(f\left(u_{\mu}\right)-f\left(u_{\tau}\right), \widetilde{U}_{t}\right)_{1}+\left(g\left(v_{\mu}\right)-g\left(v_{\tau}\right), \widetilde{V}_{t}\right)_{2} \leq \\
\max \left\{c_{2}, c_{4}\right\}\left(\left\|u_{\mu}\right\|_{L^{(p-1) n}\left(\Omega_{1}\right)}^{p-1}+\left\|u_{\tau}\right\|_{L^{(p-1) n}\left(\Omega_{1}\right)}^{p-1}+\left\|v_{\mu}\right\|_{L^{(p-1) n}\left(\Omega_{2}\right)}^{p-1}+\left\|v_{\tau}\right\|_{L^{(p-1) n}\left(\Omega_{2}\right)}^{p-1}\right) \times \\
\times\left(\|\tilde{u}\|_{L^{2 n /(n-2)}\left(\Omega_{1}\right)}\left\|\widetilde{U}_{t}\right\|_{1}+\|\tilde{v}\|_{L^{2 n /(n-2)}\left(\Omega_{2}\right)}\left\|\widetilde{V}_{t}\right\|_{2}\right) \leq \\
\leq \max \left\{c_{2}, c_{4}\right\} C_{4} C_{5}\left(\left\|\nabla u_{\mu}\right\|_{1}^{p-1}+\left\|\nabla u_{\tau}\right\|_{1}^{p-1}+\left\|\nabla v_{\mu}\right\|_{2}^{p-1}+\left\|\nabla v_{\tau}\right\|_{2}^{p-1}\right) \times \\
\times\left(\|\nabla \tilde{u}\|_{1}+\|\nabla \tilde{v}\|_{2}\right)\left(\left\|\widetilde{U}_{t}\right\|_{1}+\left\|\widetilde{V}_{t}\right\|_{2}\right) .
\end{gathered}
$$

Using (3.50) and (2.5) in (3.47) we obtain

$$
\begin{gathered}
\left\|\widetilde{U}_{t}\right\|_{1}^{2}+\|\nabla \widetilde{U}\|_{1}^{2}+\left\|\widetilde{V}_{t}\right\|_{2}^{2}+\|\nabla \widetilde{V}\|_{2}^{2}+\left\|\sqrt{M} \widetilde{\delta}_{t}\right\|_{\Gamma_{2}}^{2}+ \\
+\|\sqrt{K} \widetilde{\delta}\|_{\Gamma_{2}}^{2}+2 \int_{0}^{t}\left\|\sqrt{D} \widetilde{\delta}_{t}\right\|_{\Gamma_{2}}^{2} d s \leq \\
\leq\left\|\widetilde{U}_{1}\right\|_{1}^{2}+\left\|\nabla \widetilde{U}_{0}\right\|_{1}^{2}+\left\|\widetilde{V}_{1}\right\|_{2}^{2}+\left\|\nabla \widetilde{V}_{0}\right\|_{2}^{2}+\left\|\sqrt{M} \widetilde{\delta}_{1}\right\|_{\Gamma_{2}}^{2}+\left\|\sqrt{K} \widetilde{\delta}_{0}\right\|_{\Gamma_{2}}^{2}+ \\
+2 \max \left\{c_{2}, c_{4}\right\} C_{4} C_{5} \int_{0}^{t}\left(\left\|\nabla u_{\mu}\right\|_{1}^{p-1}+\left\|\nabla u_{\tau}\right\|_{1}^{p-1}+\left\|\nabla v_{\mu}\right\|_{2}^{p-1}+\left\|\nabla v_{\tau}\right\|_{2}^{p-1}\right) \times \\
\times\left(\|\nabla \tilde{u}\|_{1}+\|\nabla \tilde{v}\|_{2}\right)\left(\left\|\widetilde{U}_{t}\right\|_{1}+\left\|\widetilde{V}_{t}\right\|_{2}\right) d s .
\end{gathered}
$$

By (2.1) the last inequality gives

$$
\begin{gathered}
\left\|\widetilde{U}_{t}\right\|_{1}^{2}+\|\nabla \widetilde{U}\|_{1}^{2}+\left\|\widetilde{V}_{t}\right\|_{2}^{2}+\|\nabla \widetilde{V}\|_{2}^{2}+\left\|\widetilde{\delta}_{t}\right\|_{\Gamma_{2}}^{2}+ \\
+\|\widetilde{\delta}\|_{\Gamma_{2}}^{2}+\int_{0}^{t}\left\|\widetilde{\delta}_{t}\right\|_{\Gamma_{2}}^{2} d s \leq \frac{1}{C_{6}}\left(\left\|\widetilde{U}_{1}\right\|_{1}^{2}+\left\|\nabla \widetilde{U}_{0}\right\|_{1}^{2}+\left\|\widetilde{V}_{1}\right\|_{2}^{2}+\left\|\nabla \widetilde{V}_{0}\right\|_{2}^{2}+\right. \\
\left.+\max _{x \in \bar{\Gamma}_{2}} M(x)\left\|\widetilde{\delta}_{1}\right\|_{\Gamma_{2}}^{2}+\max _{x \in \bar{\Gamma}_{2}} K(x)\left\|\widetilde{\delta}_{0}\right\|_{\Gamma_{2}}^{2}\right)+
\end{gathered}
$$




$$
+\frac{K_{1}}{C_{6}} \int_{0}^{t}\left(\|\nabla \tilde{u}\|_{1}+\|\nabla \tilde{v}\|_{2}\right)\left(\left\|\widetilde{U}_{t}\right\|_{1}+\left\|\widetilde{V}_{t}\right\|_{2}\right) d s,
$$

where $C_{6}=\min \left\{1, m_{0}, 2 d_{0}, k_{0}\right\}, \min _{x \in \bar{\Gamma}_{2}} M(x)=m_{0}>0, \min _{x \in \bar{\Gamma}_{2}} D(x)=d_{0}>0$, $\min K(x)=k_{0}>0$ and $K_{1}$ is a positive constant depending only on $\Omega_{1}, \Omega_{2}, p$, $x \in \bar{\Gamma}_{2}$

T. The Gronwall's lemma guarantees that:

$$
\begin{gathered}
\|(\widetilde{U}, \widetilde{V}, \widetilde{\delta})\|_{Z_{T}}^{2} \leq K_{2}\left(\left\|\widetilde{U}_{1}\right\|_{1}^{2}+\left\|\nabla \widetilde{U}_{0}\right\|_{1}^{2}+\left\|\widetilde{V}_{1}\right\|_{2}^{2}+\left\|\nabla \widetilde{V}_{0}\right\|_{2}^{2}+\right. \\
\left.+\max _{x \in \bar{\Gamma}_{2}} M(x)\left\|\widetilde{\delta}_{1}\right\|_{\Gamma_{2}}^{2}+\max _{x \in \bar{\Gamma}_{2}} K(x)\left\|\widetilde{\delta}_{0}\right\|_{\Gamma_{2}}^{2}\right)+K_{3} T\|(\widetilde{u}, \widetilde{v}, \widetilde{\delta})\|_{Z_{T}}^{2},
\end{gathered}
$$

where $K_{2}, K_{3}$ are positive constants depending only on $\Omega_{1}, \Omega_{2}, p, T$.

Since $\left\{u_{\mu}^{0}\right\}$ is a converging sequence in $H_{\Gamma_{1}}^{1}\left(\Omega_{1}\right),\left\{v_{\mu}^{0}\right\}$ is a converging sequence in $H^{1}\left(\Omega_{2}\right),\left\{u_{\mu}^{1}\right\}$ is a converging sequence in $L^{2}\left(\Omega_{1}\right),\left\{v_{\mu}^{1}\right\}$ is a converging sequence in $L^{2}\left(\Omega_{2}\right),\left\{\delta_{\mu}^{0}\right\}$ is a converging sequence in $L^{2}\left(\Gamma_{2}\right)$ and $\left\{\delta_{\mu}^{1}\right\}$ is a converging sequence in $L^{2}\left(\Gamma_{2}\right)$, we conclude that $\left(U_{\mu}, V_{\mu}, \delta_{\mu}\right)$ is a Cauchy sequence in $Z_{T}$. Thus $\left(U_{\mu}, V_{\mu}, \delta_{\mu}\right)$ converges to the limit $(U, V, \delta) \in Z_{T}$. By the same procedure used by Georgiev and Todorova in [21] we can prove that this limit is a solution of the problem (3.44). Lemma 3.2 is proved.

Proof of Theorem 2.1. For $T>0$, we define the convex closed subset of $Z_{T}:$

$$
\begin{gathered}
X_{T}=\left\{(U, V, \delta) \in Z_{T}:\left.U\right|_{t=0}=u_{0},\left.V\right|_{t=0}=v_{0},\left.U_{t}\right|_{t=0}=u_{1},\left.V_{t}\right|_{t=0}=v_{1}\right. \\
\left.\left.\delta\right|_{t=0}=\delta_{0},\left.\delta_{t}\right|_{t=0}=\delta_{1}\right\} .
\end{gathered}
$$

For $R>0$ let us denote

$$
B_{R}\left(X_{T}\right)=\left\{(U, V, \delta) \in X_{T}:\|(U, V, \delta)\|_{Z_{T}} \leq R\right\} .
$$

Then, Lemma 3.2 implies that for any $(u, v, \delta) \in X_{T}$, we define $(U, V, \delta)=$ $\Phi(u, v, \delta)$ as a unique solution of problem (3.44) corresponding to $(u, v, \delta)$.

$\Phi$ is a contractive map satisfying $\Phi\left(B_{R}\left(X_{T}\right)\right) \subset B_{R}\left(X_{T}\right)$. Indeed, let $(u, v, \delta) \in$ $B_{R}\left(X_{T}\right)$ and $(U, V, \delta)=\Phi(u, v, \delta)$. Then for all $t \in(0, T)$ (as in the proof of Lemma 3.2) we have

$$
\begin{gathered}
C_{6}\left(\left\|U_{t}\right\|_{1}^{2}+\|\nabla U\|_{1}^{2}+\left\|V_{t}\right\|_{2}^{2}+\|\nabla V\|_{2}^{2}+\left\|\delta_{t}\right\|_{\Gamma_{2}}^{2}+\right. \\
\left.+\|\delta\|_{\Gamma_{2}}^{2}+\int_{0}^{t}\left\|\delta_{t}\right\|_{\Gamma_{2}}^{2} d s\right)+2 \int_{0}^{t}\left(\rho\left(U_{t}\right), U_{t}\right)_{\Gamma_{2}} d s+2 \int_{0}^{t} \int_{\Omega_{1}}\left|U_{t}\right|^{q_{1}+1} d x d s+ \\
+2 \int_{0}^{t} \int_{\Omega_{2}}\left|V_{t}\right|^{q_{2}+1} d x d s \leq\left\|U_{1}\right\|_{1}^{2}+\left\|\nabla U_{0}\right\|_{1}^{2}+\left\|V_{1}\right\|_{2}^{2}+\left\|\nabla V_{0}\right\|_{2}^{2}+ \\
+\max _{x \in \bar{\Gamma}_{2}} M(x)\left\|\delta_{1}\right\|_{\Gamma_{2}}^{2}+\max _{x \in \bar{\Gamma}_{2}} K(x)\left\|\delta_{0}\right\|_{\Gamma_{2}}^{2}+2 \int_{0}^{t} \int_{\Omega_{1}} f(u) U_{t} d x d s+2 \int_{0}^{t} \int_{\Omega_{2}} g(v) V_{t} d x d s .
\end{gathered}
$$


By Hölder's inequality, the condition (2.2) and the facts that $\rho(s) s \geq 0(\forall s \in$ $(-\infty,+\infty))$ and $(u, v, \delta) \in B_{R}\left(X_{T}\right)$ we get

$$
\begin{gathered}
C_{6}\left(\left\|U_{t}\right\|_{1}^{2}+\|\nabla U\|_{1}^{2}+\left\|V_{t}\right\|_{2}^{2}+\|\nabla V\|_{2}^{2}+\left\|\delta_{t}\right\|_{\Gamma_{2}}^{2}+\right. \\
\left.+\|\delta\|_{\Gamma_{2}}^{2}+\int_{0}^{t}\left\|\delta_{t}\right\|_{\Gamma_{2}}^{2} d s\right)+2 \int_{0}^{t} \int_{\Omega_{1}}\left|U_{t}\right|^{q_{1}+1} d x d s+2 \int_{0}^{t} \int_{\Omega_{2}}\left|V_{t}\right|^{q_{2}+1} d x d s \leq \\
\leq\left\|U_{1}\right\|_{1}^{2}+\left\|\nabla U_{0}\right\|_{1}^{2}+\left\|V_{1}\right\|_{2}^{2}+\left\|\nabla V_{0}\right\|_{2}^{2}+ \\
+\max _{x \in \bar{\Gamma}_{2}} M(x)\left\|\delta_{1}\right\|_{\Gamma_{2}}^{2}+\max _{x \in \bar{\Gamma}_{2}} K(x)\left\|\delta_{0}\right\|_{\Gamma_{2}}^{2}+2 C R^{p} \int_{0}^{t}\left(\left\|U_{t}\right\|_{1}+\left\|V_{t}\right\|_{2}\right) d s
\end{gathered}
$$

where $C$ is a positive constant depending only on $\Omega_{1}, \Omega_{2}, p, T$. This leads to

$$
\begin{array}{r}
\|(U, V, \delta)\|_{Z_{T}}^{2} \leq C_{6}^{-1}\left(\left\|U_{1}\right\|_{1}^{2}+\left\|\nabla U_{0}\right\|_{1}^{2}+\left\|V_{1}\right\|_{2}^{2}+\left\|\nabla V_{0}\right\|_{2}^{2}+\right. \\
\left.+\max _{x \in \bar{\Gamma}_{2}} M(x)\left\|\delta_{1}\right\|_{\Gamma_{2}}^{2}+\max _{x \in \bar{\Gamma}_{2}} K(x)\left\|\delta_{0}\right\|_{\Gamma_{2}}^{2}\right)+K_{4} R^{p} T\|(U, V, \delta)\|_{Z_{T}},
\end{array}
$$

where $K_{4}$ - a positive constant which is independent on $R$. Using Young's inequality in the last term on the right-hand side of (3.51), we get

$$
\begin{gathered}
\|(U, V, \delta)\|_{Z_{T}}^{2} \leq C_{6}^{-1}\left(\left\|U_{1}\right\|_{1}^{2}+\left\|\nabla U_{0}\right\|_{1}^{2}+\left\|V_{1}\right\|_{2}^{2}+\left\|\nabla V_{0}\right\|_{2}^{2}+\right. \\
\left.+\max _{x \in \bar{\Gamma}_{2}} M(x)\left\|\delta_{1}\right\|_{\Gamma_{2}}^{2}+\max _{x \in \bar{\Gamma}_{2}} K(x)\left\|\delta_{0}\right\|_{\Gamma_{2}}^{2}\right)+T R^{p}\left(\frac{T R^{p}}{2} K_{4}^{2}+\frac{1}{2 T R^{p}}\|(U, V, \delta)\|_{Z_{T}}^{2}\right) .
\end{gathered}
$$

Hence, we obtain

$$
\begin{gathered}
\frac{1}{2}\|(U, V, \delta)\|_{Z_{T}}^{2} \leq C_{6}^{-1}\left(\left\|U_{1}\right\|_{1}^{2}+\left\|\nabla U_{0}\right\|_{1}^{2}+\left\|V_{1}\right\|_{2}^{2}+\left\|\nabla V_{0}\right\|_{2}^{2}+\right. \\
\left.+\max _{x \in \bar{\Gamma}_{2}} M(x)\left\|\delta_{1}\right\|_{\Gamma_{2}}^{2}+\max _{x \in \bar{\Gamma}_{2}} K(x)\left\|\delta_{0}\right\|_{\Gamma_{2}}^{2}\right)+\frac{1}{2} R^{2 p} T^{2} K_{4}^{2} .
\end{gathered}
$$

By choosing $R$ large enough so that

$$
\begin{gathered}
C_{6}^{-1}\left(\left\|U_{1}\right\|_{1}^{2}+\left\|\nabla U_{0}\right\|_{1}^{2}+\left\|V_{1}\right\|_{2}^{2}+\left\|\nabla V_{0}\right\|_{2}^{2}+\right. \\
\left.+\max _{x \in \bar{\Gamma}_{2}} M(x)\left\|\delta_{1}\right\|_{\Gamma_{2}}^{2}+\max _{x \in \bar{\Gamma}_{2}} K(x)\left\|\delta_{0}\right\|_{\Gamma_{2}}^{2}\right) \leq \frac{1}{4} R^{2},
\end{gathered}
$$

then $T$ sufficiently small so that $R^{2 p} T^{2} K_{4}^{2} \leq \frac{1}{2} R^{2}$, from (3.52) we obtain: $(U, V, \delta)$ $\in B_{R}\left(X_{T}\right)$.

Next, we verify that $\Phi$ is a contraction mapping. To this end, we set $\widetilde{u}=u-\bar{u}$, $\widetilde{v}=v-\bar{v}, \widetilde{U}=U-\bar{U}, \widetilde{V}=V-\bar{V}$ and $\widetilde{\delta}=\delta-\bar{\delta}$, where $(U, V, \delta)=\Phi(u, v, \delta)$ 
and $(\bar{U}, \bar{V}, \bar{\delta})=\Phi(\bar{u}, \bar{v}, \bar{\delta})$. It is straightforward to verify that $(\widetilde{U}, \widetilde{V}, \widetilde{\delta})$ satisfies

$$
\left\{\begin{array}{c}
\widetilde{U}_{t t}-\Delta \widetilde{U}+\left|U_{t}\right|^{q_{1}-1} U_{t}-\left|\bar{U}_{t}\right|^{q_{1}-1} \bar{U}_{t}=f(u)-f(\bar{u}) \text { in } \Omega_{1} \times(0, T), \\
\widetilde{V}_{t t}-\Delta \widetilde{V}+\left|V_{t}\right|^{q_{2}-1} V_{t}-\left|\bar{V}_{t}\right|^{q_{2}-1} \bar{V}_{t}=g(v)-g(\bar{v}) \text { in } \Omega_{2} \times(0, T), \\
M \widetilde{\delta}_{t t}+D \widetilde{\delta}_{t}+K \widetilde{\delta}=-\widetilde{U}_{t} \text { on } \Gamma_{2} \times(0, T), \\
\widetilde{U}=0 \text { on } \Gamma_{1} \times(0, T), \\
\widetilde{U}=\widetilde{V}, \frac{\partial \widetilde{U}}{\partial \nu}-\frac{\partial \widetilde{V}}{\partial \nu}+\rho\left(U_{t}\right)-\rho\left(\bar{U}_{t}\right)=\widetilde{\delta}_{t} \text { on } \Gamma_{2}, \\
\widetilde{U}(x, 0)=0, \widetilde{U}_{t}(x, 0)=0, x \in \Omega_{1}, \\
\widetilde{V}(x, 0)=0, \widetilde{V}_{t}(x, 0)=0, x \in \Omega_{2}, \\
\widetilde{\delta}(x, 0)=0, \widetilde{\delta}_{t}(x, 0)=0, x \in \Gamma_{2} .
\end{array}\right.
$$

Multiplying the first equation by $2 \widetilde{U}_{t}$, the second equation by $2 \widetilde{V}_{t}$ and the third equation by $2 \widetilde{\delta}_{t}$ in (3.53) and integrating over $(0, t) \times \Omega_{1},(0, t) \times \Omega_{2}$, and $(0, t) \times \Gamma_{2}$, respectively, we obtain

$$
\begin{gathered}
\frac{d}{d t}\left(\left\|\widetilde{U}_{t}\right\|_{1}^{2}+\|\nabla \widetilde{U}\|_{1}^{2}+\left\|\widetilde{V}_{t}\right\|_{2}^{2}+\|\nabla \widetilde{V}\|_{2}^{2}+\left\|\sqrt{M} \widetilde{\delta}_{t}\right\|_{\Gamma_{2}}^{2}+\|\sqrt{K} \widetilde{\delta}\|_{\Gamma_{2}}^{2}\right)+ \\
+2\left(\rho\left(U_{t}\right)-\rho\left(\bar{U}_{t}\right), U_{t}-\bar{U}_{t}\right)_{\Gamma_{2}}+2\left\|\sqrt{D} \widetilde{\delta}_{t}\right\|_{\Gamma_{2}}^{2}+ \\
+2\left(\left|U_{t}\right|^{q_{1}-1} U_{t}-\left|\bar{U}_{t}\right|^{q_{1}-1} \bar{U}_{t}, U_{t}-\bar{U}_{t}\right)_{1}+2\left(\left|V_{t}\right|^{q_{2}-1} V_{t}-\left|\bar{V}_{t}\right|^{q_{2}-1} V_{t}, V_{t}-\bar{V}_{t}\right)_{2}= \\
=2\left(f(u)-f(\bar{u}), \widetilde{U}_{t}\right)_{1}+2\left(g(v)-g(\bar{v}), \widetilde{V}_{t}\right)_{2} .
\end{gathered}
$$

Since

$$
\begin{gathered}
\left(\rho\left(U_{t}\right)-\rho\left(\bar{U}_{t}\right), U_{t}-\bar{U}_{t}\right)_{\Gamma_{2}} \geq 0, \\
\left(\left|U_{t}\right|^{q_{1}-1} U_{t}-\left|\bar{U}_{t}\right|^{q_{1}-1} \bar{U}_{t}, U_{t}-\bar{U}_{t}\right)_{1} \geq 0, \\
\left(\left|V_{t}\right|^{q_{2}-1} V_{t}-\left|\bar{V}_{t}\right|^{q_{2}-1} \bar{V}_{t}, V_{t}-\bar{V}_{t}\right)_{2} \geq 0, \\
\min _{x \in \bar{\Gamma}_{2}} M(x)=m_{0}>0, \min _{x \in \bar{\Gamma}_{2}} D(x)=d_{0}>0, \min _{x \in \bar{\Gamma}_{2}} K(x)=k_{0}>0
\end{gathered}
$$

and by (3.50):

$$
\begin{gathered}
\left(f(u)-f(\bar{u}), \widetilde{U}_{t}\right)_{1}+\left(g(v)-g(\bar{v}), \widetilde{V}_{t}\right)_{2} \leq \\
\leq \max \left\{c_{2}, c_{4}\right\} C_{4} C_{5}\left(\|\nabla u\|_{1}^{p-1}+\|\nabla \bar{u}\|_{1}^{p-1}+\|\nabla v\|_{2}^{p-1}+\|\nabla \bar{v}\|_{2}^{p-1}\right) \times \\
\times\left(\|\nabla \tilde{u}\|_{1}+\|\nabla \tilde{v}\|_{2}\right)\left(\left\|\widetilde{U}_{t}\right\|_{1}+\left\|\widetilde{V}_{t}\right\|_{2}\right),
\end{gathered}
$$

then from (3.54) we obtain

$$
\begin{gathered}
\left\|\widetilde{U}_{t}\right\|_{1}^{2}+\|\nabla \widetilde{U}\|_{1}^{2}+\left\|\widetilde{V}_{t}\right\|_{2}^{2}+\|\nabla \widetilde{V}\|_{2}^{2}+\left\|\widetilde{\delta}_{t}\right\|_{\Gamma_{2}}^{2}+\|\widetilde{\delta}\|_{\Gamma_{2}}^{2}+\int_{0}^{t}\left\|\widetilde{\delta}_{t}\right\|_{\Gamma_{2}}^{2} d s \leq \\
\leq \frac{2 \max \left\{c_{2}, c_{4}\right\} C_{4} C_{5}}{C_{6}} \int_{0}^{t}\left(\|\nabla u\|_{1}^{p-1}+\|\nabla \bar{u}\|_{1}^{p-1}+\|\nabla v\|_{2}^{p-1}+\|\nabla \bar{v}\|_{2}^{p-1}\right) \times \\
\times\left(\|\nabla \tilde{u}\|_{1}+\|\nabla \tilde{v}\|_{1}\right)\left(\left\|\widetilde{U}_{t}\right\|_{1}+\left\|\widetilde{V}_{t}\right\|_{2}\right) d s .
\end{gathered}
$$

Thus we have

$$
\|(\widetilde{U}, \widetilde{V}, \widetilde{\delta})\|_{Z_{T}} \leq K_{5} T R^{p-1}\|(\tilde{u}, \tilde{v}, \tilde{\delta})\|_{Z_{T}},
$$

where $K_{5}$ is a positive constant depending only on $\Omega_{1}, \Omega_{2}$ and $p$.

By choosing $T$ small enough in order to have $K_{5} T R^{p-1}<1$, the estimate (3.55) shows that $\Phi$ is a contraction. By the contraction mapping theorem we 
obtain the existence of a unique solution $(U, V, \delta)$ satisfying $(U, V, \delta)=\Phi(U, V, \delta)$. Theorem 2.1 is proved.

\section{References}

[1] A. B. Aliev, S. E. Isayeva, Exponential stability of the nonlinear transmission acoustic problem, Math. Methods Appl. Sci. 41 (16) (2018), 7055-7073.

[2] A. B. Aliev, S. E. Isayeva, Existence and nonexistence of global solutions for nonlinear transmission acoustic problem, Turkish J. Math. 42 (2018), 3211-3231.

[3] A. B. Aliev, E. H. Mammadhasanov, Well-posedness of initial boundary value problem on longitudind impact on a composite linear viscoelastic bar, Math. Methods Appl. Sci. 40(14) (2017), 5380-5390.

[4] J. J. Bae, Nonlinear transmission problem for wave equation with boundary condition of memory type, Acta, Appl. Math. 110(2) (2010), 907-919.

[5] W. D. Bastos, C. A. Raposo, Transmission problem for waves with frictional damping, Electron. J. Differential Equations 60 (2007), 1-10.

[6] J. T. Beale, S. I. Rosencrans, Acoustic boundary conditions, Bull. Amer. Math. Soc. 80(6) (1974), 1276-1278.

[7] J. T. Beale, Spectral properties of an acoustic boundary condition, Indiana Univ. Math. J. 25(9) (1976), 895-917.

[8] J. T. Beale, Acoustic scattering from locally reacting surfaces, Indiana Univ. Math. J. 26 (1977), 199-222.

[9] Y. Boukhatem, B. Benabderrahmane, Existence and decay of solutions for a viscoelastic wave equation with acoustic boundary conditions, Nonlinear Analysis $\mathbf{9 7}$ (2014), 191-209.

[10] Y. Boukhatem, B. Benabderrahmane, Polynomial Decay and Blow Up of Solutions for Variable Coefficients Viscoelastic Wave Equation with Acoustic Boundary Conditions, Acta Mathematica Sinica, English series, 32 (2) (2016), 153-174.

[11] H. Brezis, Analyse fonctionelle thẽorie et applications, Masson, Paris, 1983.

[12] A. T. Cousin, C. L. Frota, N. A. Larkin, On a system of Klein-Gordon type equations with acoustic boundary conditions, J. Math. Appl. 293 (2004), 293-309.

[13] R. Dautray, J. L. Lions, Analyse et Calcul Numerique pour les Sciences et les Techniques, Tome 1, Masson, Paris, 1984.

[14] C. L. Frota, J. A. Goldstein, Some wave equations with acoustic boundary conditions, J. Differential Equations 164 (2000), 92-109.

[15] C. L. Frota, A. T. Cousin, N. A. Larkin, Global solvability and asymptotic behaviour of a hyperbolic problem with acoustic boundary conditions, Funkcial. Ekvac. 44 (3) (2001), 471-485.

[16] C. I. Frota, N. A. Larkin, Uniform stabilization for a hyperbolic equation with acoustic boundary conditions in simple connected domains, Progr. Nonlinear Differential Equations Appl. 66(2005), 297-312.

[17] C. L. Frota, A. Vicente, A hyperbolic system of Klein-Gordon type with acoustic boundary conditions, Int. J.Pure Appl. Math. 47(2) (2008), 185-198.

[18] C. L. Frota, L. A. Medeyros, A. Vicente, A mixed problem for semilinear wave equations with acoustic boundary conditions in domains with non-locally reacting boundary, Electron. J. Differential Equations 2014(243) (2014), 1-14.

[19] C. G. Gal, G. R. Goldstein, J. A. Goldstein, Oscillatory boundary conditions for acoustic wave equations, J. Evol. Equ. 3(4) (2003), 623-635.

[20] Y. Gao, J. Liang, T. J. Xiao; Observability inequality and decay rate for wave equations with nonlinear boundary conditions, Electron. J. Differential Equations 2017 (161) (2017), 1-12. 
[21] V. Georgiev, G. Todorova, Existence of a global solution of the wave equation with nonlinear damping and source term, J. Differential Equations 109 (1994) 295-308.

[22] P. J. Graber, Wave equation with porous nonlinear acoustic boundary conditions generates a well-posed dynamical system, Nonlinear Anal. 73 (2010), 3058-3068.

[23] P. J. Graber, Strong stability and uniform decay of silutions to a wave equation with semilinear porous acoustic boundary conditions, Nonlinear Anal. 74 (2011), 3137-3148.

[24] P. J. Graber, B. Said-Houari, On the wave equation with semilinear porous acoustic boundary conditions, J. Differential Equations 252 (2012), 4898-4941.

[25] J. M. Jeong, J. Y. Park, Y. H. Kang, Global nonexistence of solutions for a quasilinear wave equation with acoustic boundary conditions, Boundary Value Problems 2017 (42) (2017), 1-10.

[26] J. L. Lions, Quelques mẽthodes de rẽsolution des problẽmes aux limites non linẽaires, Dunod Gaulthier-Villars, Paris, 1969.

[27] J. L. Lions, E. Magenes, Non homogeneous boundary value problems and applications I, Springer Verlag, Berlin, 1972.

[28] W. Liu, G. Williams, The Exponential Stability of the Problem of Transmission of the Wave Equation, Bull. Austral. Math. Soc. 57 (1998), 305-327.

[29] P. M. Morse, K. U. Ingard, Theoretical Acoustic, MaGraw-Hill, 1968.

[30] D. Mugnolo, Abstract wave equations with acoustic boundary conditions, Math. Nachr. 279(3) (2006), 299-318.

[31] J. E. Muñoz Rivera, H. Portillo Oquendo, The transmission problem of viscoelastic waves, Acta Applicandae Mathematicae 60 (2000), 1-21.

[32] J. Y. Park, T. G. Ha, Well-posedness and uniform decay rates for the Klein-Gordon equation with damping term and acoustic boundary conditions, J. Math. Phys. 50 (1) 013506 (2009), 1-18.

[33] J. Y. Park, S. H. Park, Decay rate estimates for wave equations of memory type with acoustic boundary conditions, Nonlinear Analysis 74 (2011), 993-998.

[34] A. Vicente, Wave equation with acoustic/memory boundary conditions, Bol. Soc. Parana. Mat. (3) 27 (1) (2009), 29-39.

Sevda E. Isayeva

Baku State University, Baku, Azerbaijan.

E-mail address: isayevasevda@rambler.ru

Received: November 12, 2020; Accepted: April 30, 2021 